Supporting Materials for

\title{
Evaluation of Asymmetric Hydrogenation Ligands in Asymmetric Hydroformylation Reactions. Highly Enantioselective Ligands Based on Bis-Phosphacycles.
}

\author{
Alex T. Axtell, Jerzy Klosin,* \\ Corporate R\&D, The Dow Chemical Company, 1776 Building, Midland, MI 48674 \\ jklosin@dow.com
}

Khalil A. Abboud

Department of Chemistry, University of Florida, Gainesville, FL 32611

Contents

1) Structures of some ligands investigated in this study

2) Asymmetric hydroformylation results obtained with ligands shown in Chart 1.

3) NMR spectra of $[(S, S, R, R)$-TangPhos $] \mathrm{Rh}($ acac) $(19)$

4) NMR spectra of $\left.\left[((S, S, R, R)-\text { TangPhos })_{2}\right] \mathrm{Rh}\right]^{+}[\mathrm{acac}]^{-}$

5) NMR spectra of $[\mathrm{N}(n-\mathrm{Bu})]^{+}[\mathrm{acac}]^{-}$

6) NMR spectra of reactions between 19 and $\mathrm{Rh}(\mathrm{CO})_{2}$ (acac) and $(S, S, R, R)$-TangPhos

7) X-ray data table and bond lengths and angle tables for $[(S, S, R, R)$-TangPhos $] \mathrm{Rh}(\mathrm{acac})(\mathbf{1 9})$

8) X-ray data table and bond lengths and angle tables for $\left.\left[((S, S, R, R) \text {-TangPhos })_{2}\right] \mathrm{Rh}\right]^{+}[\mathrm{acac}]^{-}$ 
Chart 1. Some of asymmetric ligands screened in this study

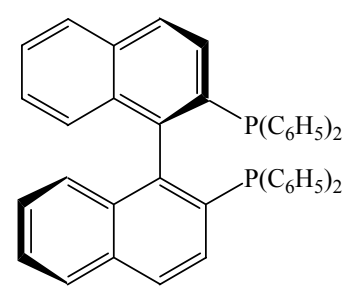

(R)-BINAP (20)

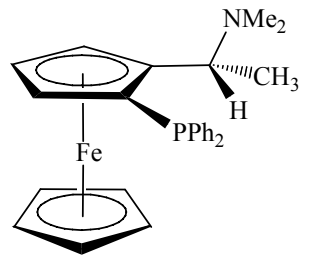

$\mathrm{C}_{26} \mathrm{H}_{28} \mathrm{FeNP}$ (23)

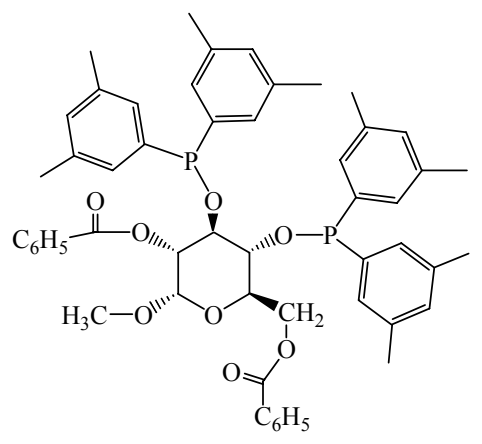

$\alpha$ D-CARBOPHOS (27)

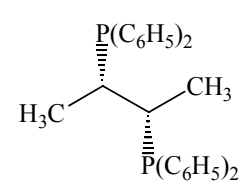

(S,S)-CHIRAPHOS (21)

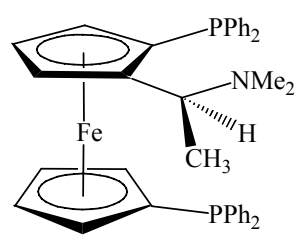

$\mathrm{C}_{38} \mathrm{H}_{37} \mathrm{FeNP}_{2}$ (24)

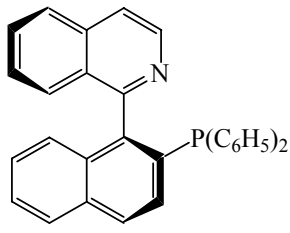

(S)-QUINAP (28)

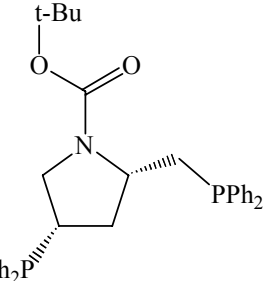

(S,S)-BPPM (22)

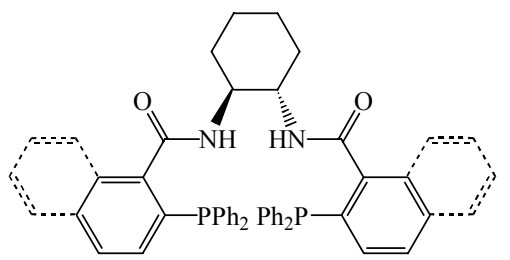

(1R,2R)-Trost Ligand (Phenyl) (25) $(1 S, 2 S)$-Trost Ligand (Naphthyl) (26)

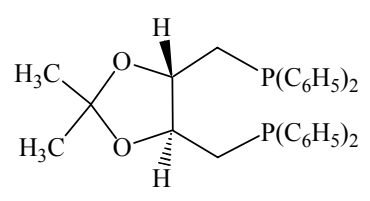

(S,S)-DIOP (29) 


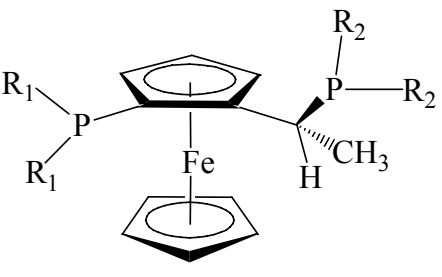

Josiphos

$30-\mathrm{R} 1=\mathrm{Cy}, \mathrm{R} 2=\mathrm{Cy}$

$31-\mathrm{R} 1=3,5-$ di- $\mathrm{CF}_{3}-\mathrm{Ph}, \mathrm{R} 2=\mathrm{Cy}$

$32-\mathrm{R} 1=3,5-$ di- $\mathrm{CH}_{3}-4-\mathrm{OMe}-\mathrm{Ph}, \mathrm{R} 2=\mathrm{Cy}$

$33-\mathrm{R} 1=3,5-\mathrm{di}-\mathrm{CF}_{3}-\mathrm{Ph}, \mathrm{R} 2=3,5-\mathrm{di}-\mathrm{CH}_{3}-\mathrm{Ph}$

$34-\mathrm{R} 1=\mathrm{Cy}, \mathrm{R} 2=t-\mathrm{Bu}$

$35-\mathrm{R} 1=2$-furyl, R2 = 3,5-di- $\mathrm{CH}_{3}-\mathrm{Ph}$

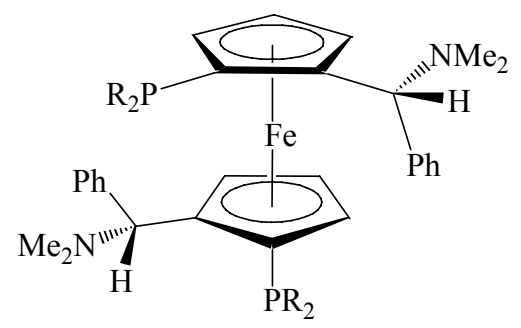

Mandyphos

$39-\mathrm{R}=\mathrm{Ph}$
$40-\mathrm{R}=\mathrm{Cy}$
$41-\mathrm{R}=3,5-$ di- $\mathrm{CH}_{3}-4-\mathrm{OMe}-\mathrm{Ph}$
$42-\mathrm{R}=3,5-\mathrm{di}-\mathrm{CH}_{3}-\mathrm{Ph}$
$43-\mathrm{R}=2-\mathrm{CH}_{3}-\mathrm{Ph}$

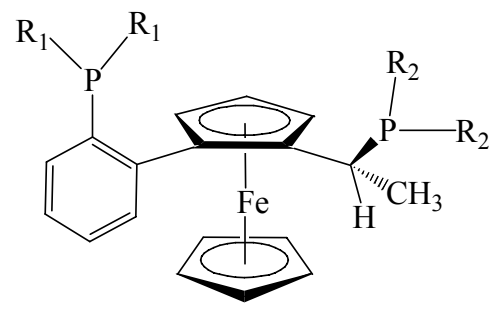

Walphos

$36-\mathrm{R} 1=\mathrm{Ph}, \mathrm{R} 2=\mathrm{Cy}$

$37-\mathrm{R} 1=\mathrm{Ph}, \mathrm{R} 2=3,5-\mathrm{di}-\mathrm{CH}_{3}-\mathrm{Ph}$

38 - R1 = 3,5-di- $\mathrm{CH}_{3}-\mathrm{Ph}, \mathrm{R} 2=3,5-\mathrm{di}-\mathrm{CH}_{3}-\mathrm{Ph}$

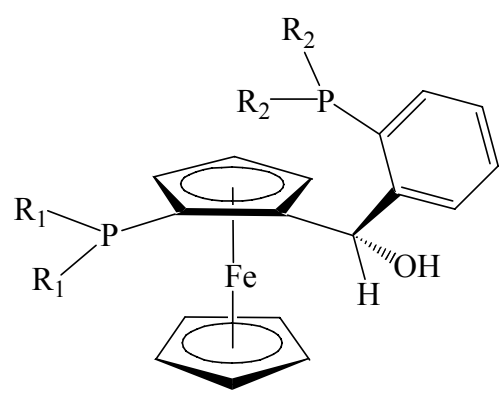

$\mathrm{C}_{47} \mathrm{H}_{40} \mathrm{~F}_{6} \mathrm{FeOP}_{2}$ (44)

$\mathrm{R} 1=3,5-\mathrm{di}-\mathrm{CH}_{3}-\mathrm{Ph}, \mathrm{R} 2=3,5-\mathrm{di}-\mathrm{CF}_{3}-\mathrm{Ph}$ 


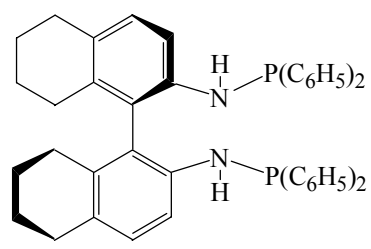

CTH-(R)-BINAM (45)

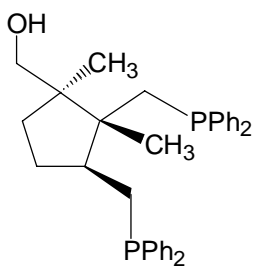

[catASium $\left.\left.{ }^{\circledR} \mathrm{I}\right)\right](\mathbf{4 8})$

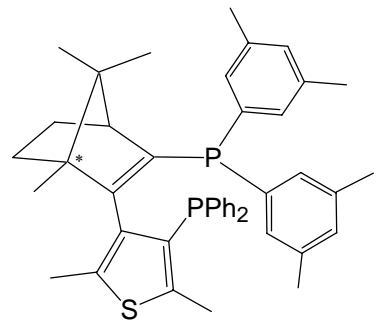

catASium ${ }^{\circledR}$ T2 (51)

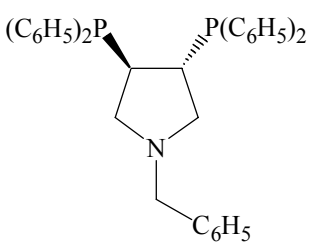

$\left[\operatorname{catASium}{ }^{\circledR} \mathrm{D}(R)\right](\mathbf{4 6})$

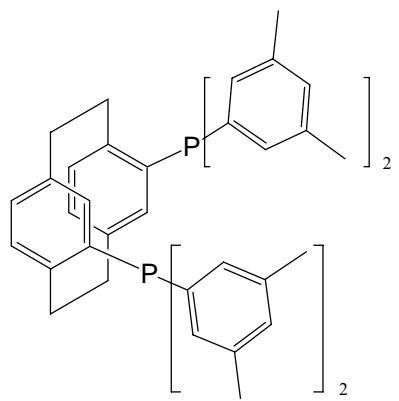

CTH-(R)-3,5-xylyl-PHANEPHOS (49)

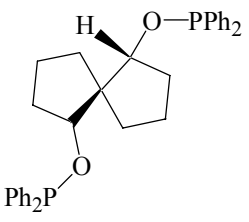

CTH-(R)-SpiroP (52)

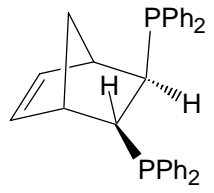

$(R, R)-N O R P H O S$ (47)

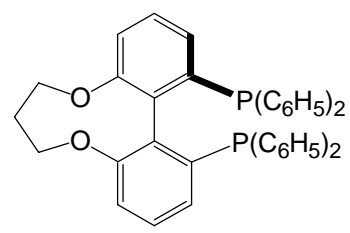

(R,)-C3-TUNEPHOS (50)

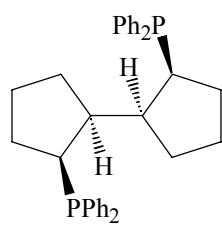

(S,S)-BICP (53) 
Table 1. Percentage conversion (conv.), branched:linear ratio (b:l), and enantioselectivity (\%ee) for hydroformylation of styrene, allyl cyanide, and vinyl acetate (olefin: $\mathrm{Rh}=3,000)$

\begin{tabular}{|c|c|c|c|c|c|c|c|c|c|c|}
\hline \multirow{2}{*}{ Entry } & \multirow{2}{*}{$\mathrm{L}$} & \multicolumn{3}{|c|}{ Styrene } & \multicolumn{3}{|c|}{ Alyl cyanide } & \multicolumn{3}{|c|}{ Vinyl acetate } \\
\hline & & conv. & $\mathrm{b}: 1$ & $\%$ ee & conv. & $\mathrm{b}: 1$ & $\%$ ee & conv. & $\mathrm{b}: 1$ & $\%$ ee \\
\hline 1 & $(R)$-Binap (20) & 8 & 10.2 & $28(S)$ & 27 & 4.2 & $6(R)$ & 18 & 9.2 & $47(S)$ \\
\hline 2 & $(S, S)$-Chiraphos $(\mathbf{2 1})$ & 5 & 15.9 & $2(R)$ & 46 & 5.4 & $0.5(S)$ & 37 & 19.3 & $2(S)$ \\
\hline 3 & $(R, R)-\mathrm{BPPM}(22)$ & 37 & 1.5 & $6(R)$ & 87 & 0.9 & $1(R)$ & 43 & 2.9 & $3(S)$ \\
\hline 4 & $\mathrm{C}_{26} \mathrm{H}_{28} \mathrm{FeNP}$ (23) & 60 & 2.5 & $4(R)$ & 96 & 2.3 & $7(R)$ & 48 & 171.2 & $9(S)$ \\
\hline 5 & $\mathrm{C}_{38} \mathrm{H}_{37} \mathrm{FeNP}_{2}(24)$ & 31 & 3.2 & $8(R)$ & 68 & 1.0 & $1(S)$ & 31 & 6.4 & $4(R)$ \\
\hline 6 & Trost Ligand (Phenyl) (25) & 5 & 3.9 & $4(R)$ & 52 & 1.6 & $6(S)$ & 18 & 15.7 & $7(R)$ \\
\hline 7 & Trost Ligand (Naphthyl) (26) & 5 & 2.8 & $24(R)$ & 23 & 1.9 & $1(R)$ & 21 & 12.6 & $7(R)$ \\
\hline 8 & $\alpha \mathrm{D}$-Carbophos (27) & 61 & 6.1 & $6(S)$ & 92 & 3.6 & $10(R)$ & 38 & 25.2 & $10(S)$ \\
\hline 9 & (S)-Quinap (28) & 18 & 3.2 & $1(S)$ & 50 & 2.2 & $1(S)$ & 24 & 37.2 & $1(R)$ \\
\hline 10 & $(S, S)-\mathrm{DIOP}(\mathbf{2 9})$ & 48 & 1.5 & $13(R)$ & 96 & 0.8 & $14(R)$ & 54 & 3.5 & $33(S)$ \\
\hline 11 & Josiphos (30) & 3 & 7.1 & $47(S)$ & 13 & 6.4 & $39(S)$ & 16 & 12.2 & $29(R)$ \\
\hline 12 & Josiphos (31) & 26 & 9.9 & $1(R)$ & 47 & 4.0 & $29(R)$ & 31 & 11.6 & $63(S)$ \\
\hline 13 & Josiphos (32) & 13 & 15.3 & $31(R)$ & 48 & 7.3 & $43(R)$ & 18 & 141.4 & $59(S)$ \\
\hline 14 & Josiphos (33) & 60 & 24.2 & $55(S)$ & 81 & 12 & $47(S)$ & 31 & 11.2 & $20(S)$ \\
\hline 15 & Josiphos (34) & 4 & 5.2 & $24(R)$ & 13 & 7.2 & $6(S)$ & 17 & 12.1 & $20(S)$ \\
\hline 16 & Josiphos (35) & 41 & 17.9 & $30(R)$ & 85 & 9.2 & $11(R)$ & 27 & 229.2 & $6(R)$ \\
\hline 17 & Walphos (36) & 10 & 0.8 & $48(S)$ & 49 & 2.1 & $10(S)$ & 26 & 30.8 & $47(S)$ \\
\hline 18 & Walphos (37) & 42 & 2.6 & $5(R)$ & 90 & 1.3 & $5(S)$ & 45 & 8.1 & $29(R)$ \\
\hline 19 & Walphos (38) & 43 & 2.9 & $4(R)$ & 87 & 1.4 & $4(S)$ & 46 & 14 & $17(R)$ \\
\hline 20 & Mandyphos (39) & 47 & 5.6 & $24(S)$ & 77 & 1.2 & $13(R)$ & 44 & 5.2 & $58(R)$ \\
\hline 21 & Mandyphos (40) & 5 & 1.5 & $3(S)$ & 20 & 2.6 & $25(S)$ & 20 & 10.1 & $33(S)$ \\
\hline 22 & Mandyphos (41) & 15 & 6.6 & $10(S)$ & 41 & 1.1 & $19(R)$ & 27 & 5.4 & $46(R)$ \\
\hline 23 & Mandyphos (42) & 17 & 8.3 & $13(S)$ & 42 & 1.1 & $20(R)$ & 25 & 4.7 & $51(R)$ \\
\hline 24 & Mandyphos (43) & 4 & 0.8 & 1 & 25 & 1.7 & 0 & 21 & 109.8 & 0.5 \\
\hline 25 & $\mathrm{C}_{47} \mathrm{H}_{40} \mathrm{~F}_{6} \mathrm{FeOP}_{2}$ (44) & 26 & 7.4 & $26(R)$ & 49 & 2.5 & $37(R)$ & 23 & 11.6 & $7(R)$ \\
\hline 26 & CTH- $(R)$-Binam (45) & 12 & 2.1 & $2(R)$ & 55 & 2.0 & $4(S)$ & 27 & 22.9 & $5(S)$ \\
\hline 27 & {$\left[\right.$ catASium $\left.{ }^{\circledR} \mathrm{D}(R)\right](46)$} & 24 & 18.5 & $10(S)$ & 61 & 7.1 & $1(R)$ & 24 & 372.4 & $16(S)$ \\
\hline 29 & $(R, R)$-NORPHOS (47) & 23 & 15.7 & $17(S)$ & 64 & 7.0 & $15(R)$ & 25 & 386 & $39(S)$ \\
\hline 30 & {$\left[\right.$ CatASium ${ }^{\circledR}$ I] (48) } & 19 & 4.2 & $29(S)$ & 75 & 4.7 & $56(S)$ & 22 & 7.9 & $3(R)$ \\
\hline 31 & $\begin{array}{l}\text { CTH-(R)-3,5-xylyl- } \\
\text { PHANEPHOS (49) }\end{array}$ & 27 & 8.8 & $8(S)$ & 44 & 1.7 & 0 & 28 & 9.3 & $29(R)$ \\
\hline 32 & (R)-C3-TUNEPHOS (50) & 8 & 11.0 & $19(S)$ & 24 & 5.3 & $12(R)$ & 16 & 8.8 & $56(S)$ \\
\hline 33 & {$\left[\right.$ CatASium ${ }^{\circledR}$ T2] (51) } & 12 & 8.7 & $26(S)$ & 35 & 3.9 & $7(S)$ & 22 & 21.2 & $45(S)$ \\
\hline 34 & CTH-(R)-SpiroP (52) & 40 & 2.0 & $14(S)$ & 87 & 1.4 & $2(R)$ & 42 & 7.3 & $23(S)$ \\
\hline
\end{tabular}




\begin{tabular}{|l|l|lll|lll|lll|}
35 & $(S, S)-B I C P(53)$ & 26 & 14.7 & $35(R)$ & 88 & 6.9 & $41(R)$ & 28 & 74.4 & $11(R)$ \\
\hline
\end{tabular}

${ }^{a}$ All reactions performed at $80^{\circ} \mathrm{C}$ in toluene with 150 psi $1: 1 \mathrm{CO}: \mathrm{H}_{2}$ with $\mathrm{L}: \mathrm{Rh}=2$, total substrate: $\mathrm{Rh}=$ $3000,1 \mathrm{~mL}$ of substrates and 3 hour reaction time. ${ }^{b} \mathrm{~L}: \mathrm{Rh}=1.2$, total substrate: $\mathrm{Rh}=3000,0.5 \mathrm{~mL}$ substrates. Ligands in blue gave between 50 and $70 \%$ ee whereas those in red gave enantioselectivities higher then $70 \%$. 
Figure 1. ${ }^{1} \mathrm{H}$ NMR spectrum of $[(S, S, R, R)$-TangPhos $] \mathrm{Rh}(\mathrm{acac})(\mathbf{1 9})$ in $\mathrm{C}_{6} \mathrm{D}_{6}$.

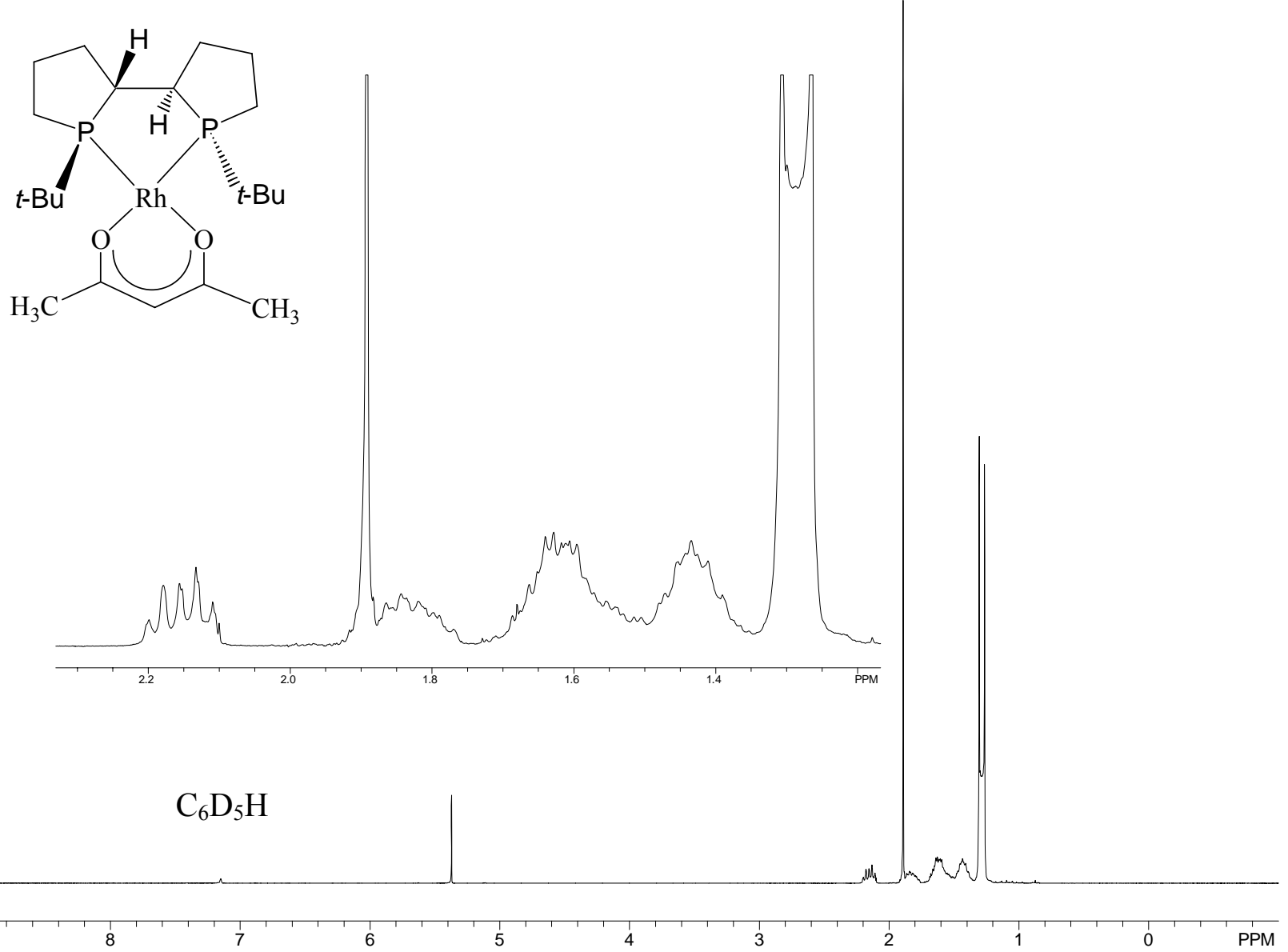


Figure 2. ${ }^{13} \mathrm{C}\left\{{ }^{1} \mathrm{H}\right\}$ and ${ }^{31} \mathrm{P}\left\{{ }^{1} \mathrm{H}\right\}$ NMR spectra of $[(S, S, R, R)$-TangPhos $] \mathrm{Rh}\left(\right.$ acac) (19) in $\mathrm{C}_{6} \mathrm{D}_{6}$.

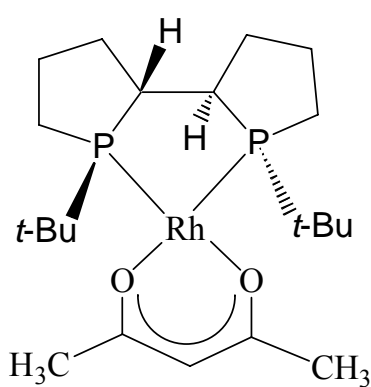
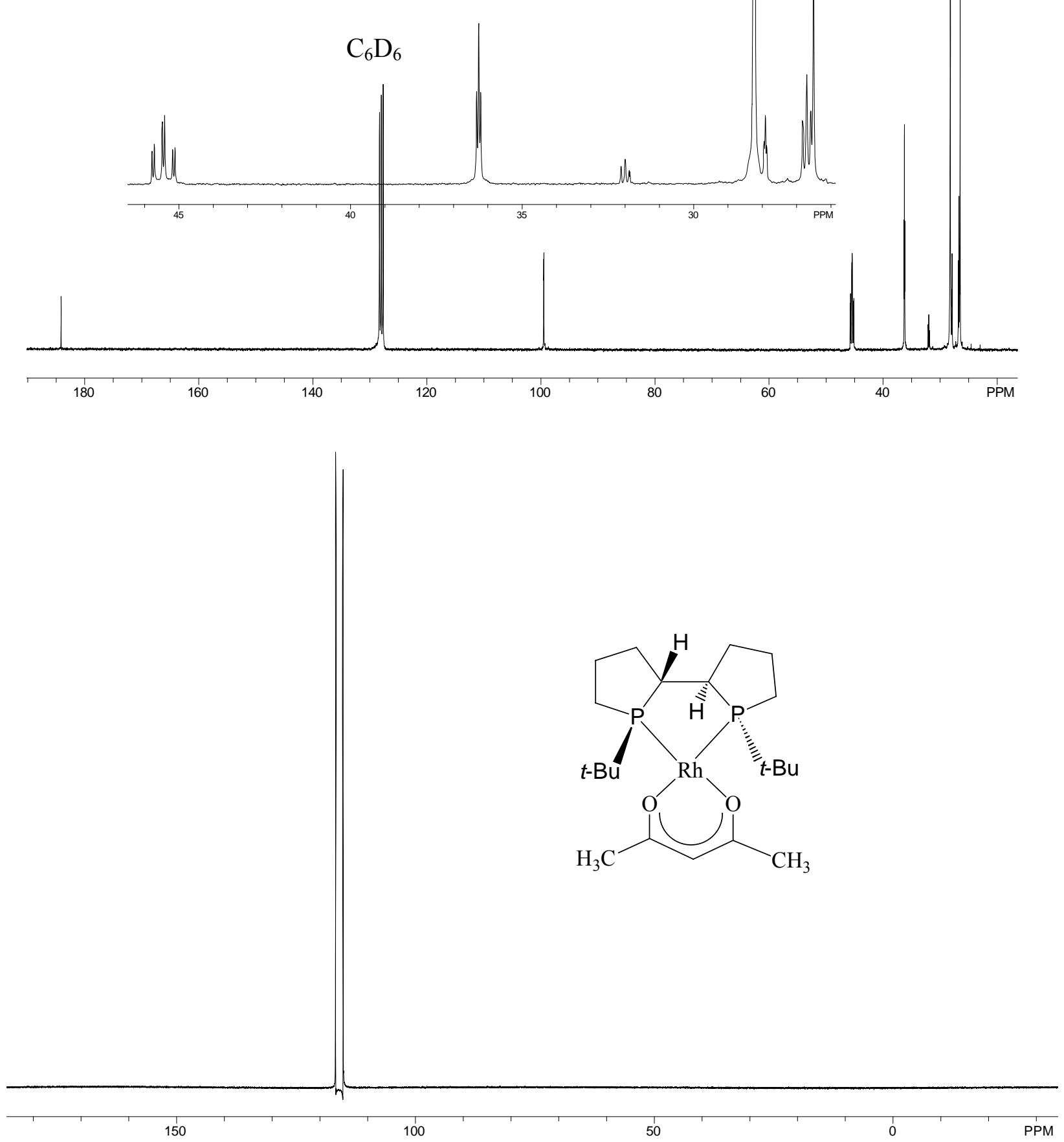
Figure 3. ${ }^{1} \mathrm{H}$ and ${ }^{31} \mathrm{P}\left\{{ }^{1} \mathrm{H}\right\} \mathrm{NMR}$ spectra of $\left.\left[((S, S, R, R) \text {-TangPhos })_{2}\right] \mathrm{Rh}\right]^{+}[\text {acac }]^{-}$in $\mathrm{C}_{6} \mathrm{D}_{5} \mathrm{Cl}$.
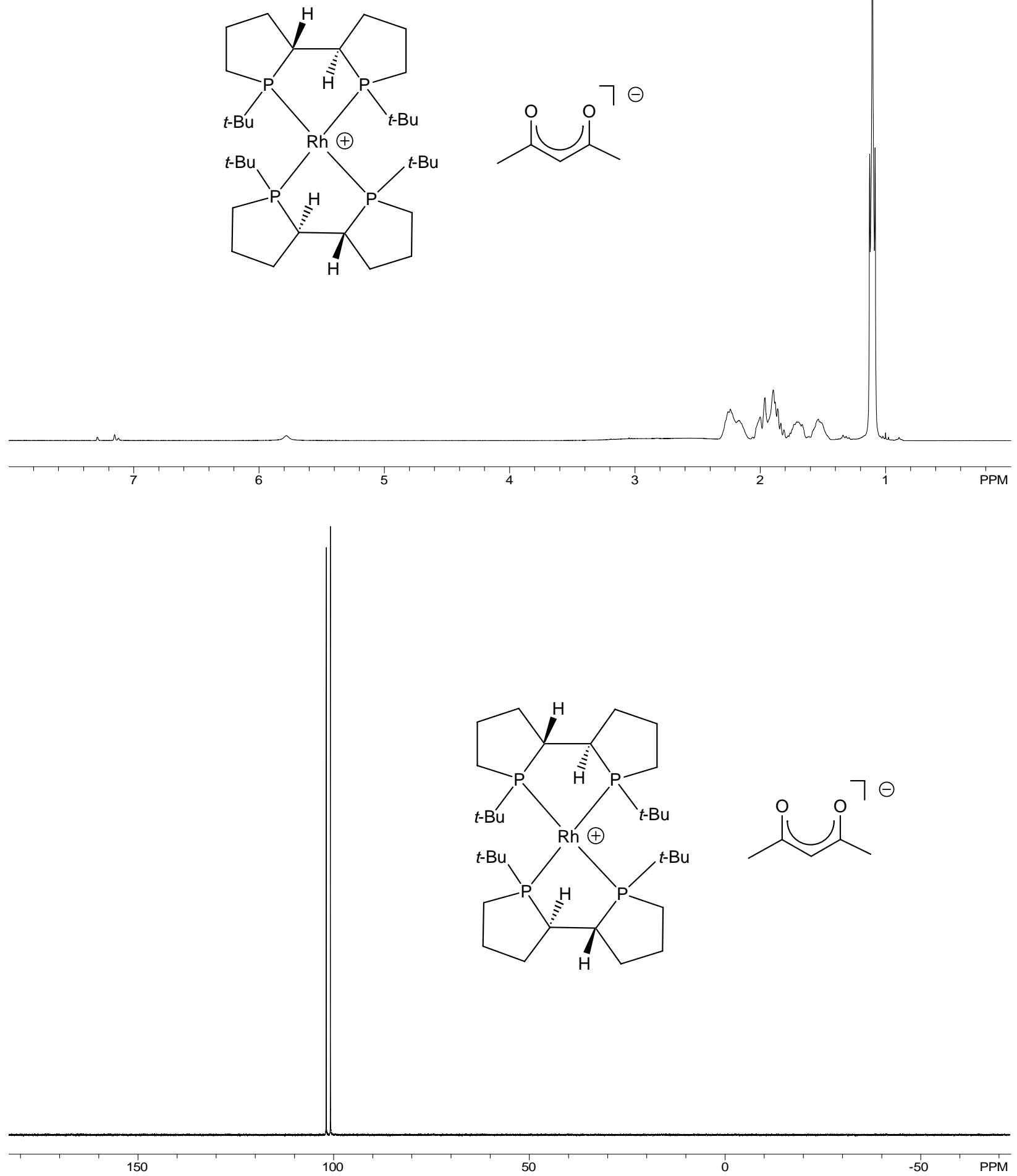
Figure $4 .{ }^{13} \mathrm{C}\left\{{ }^{1} \mathrm{H}\right\}$ and APT spectra of $\left.\left[((S, S, R, R)-\text { TangPhos })_{2}\right] \mathrm{Rh}\right]^{+}[\mathrm{acac}]^{-}$in $\mathrm{C}_{6} \mathrm{D}_{5} \mathrm{Cl}$.
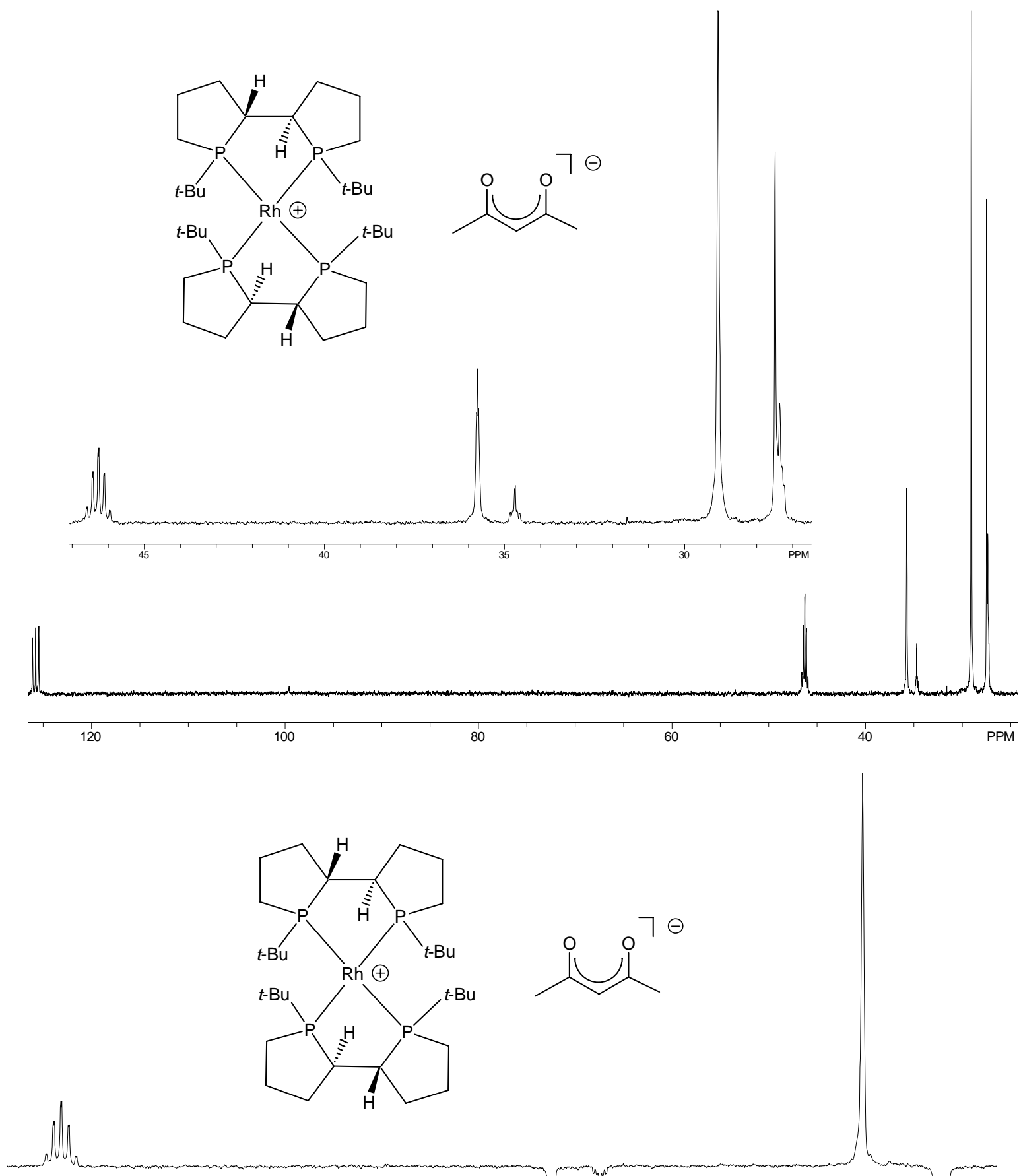
Figure 5. Mass spectrum of $\left.\left[((S, S, R, R)-\text { TangPhos })_{2}\right] \mathrm{Rh}\right]^{+}[\mathrm{acac}]^{-}$in $\mathrm{C}_{6} \mathrm{D}_{5} \mathrm{Cl}$.

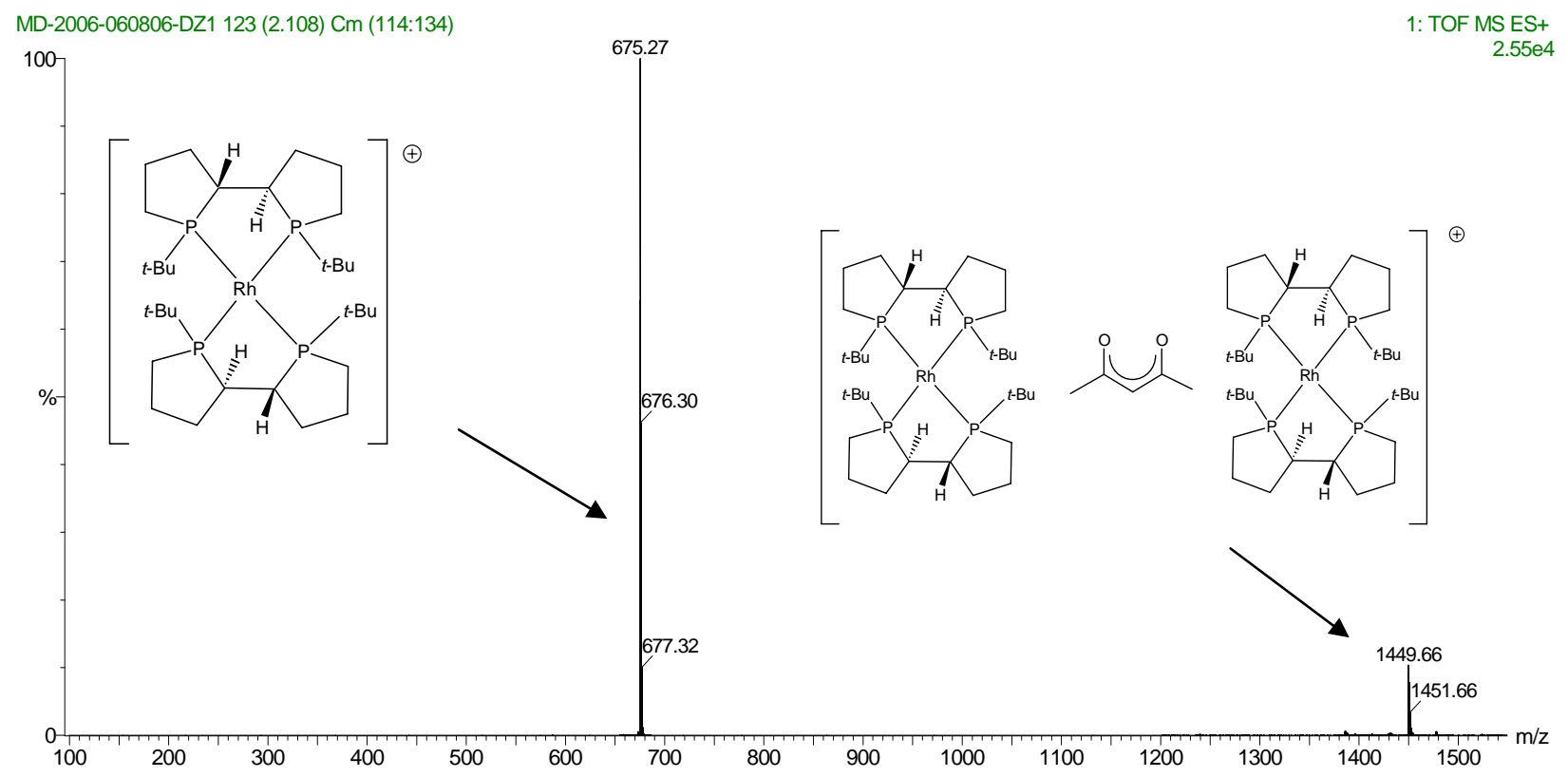


Figure 6. ${ }^{1} \mathrm{H}$ and ${ }^{13} \mathrm{C}\left\{{ }^{1} \mathrm{H}\right\} \mathrm{NMR}$ spectra of $[\mathrm{N}(n-\mathrm{Bu})]^{+}[\mathrm{acac}]^{-}$in $\mathrm{CD}_{2} \mathrm{Cl}_{2}$.
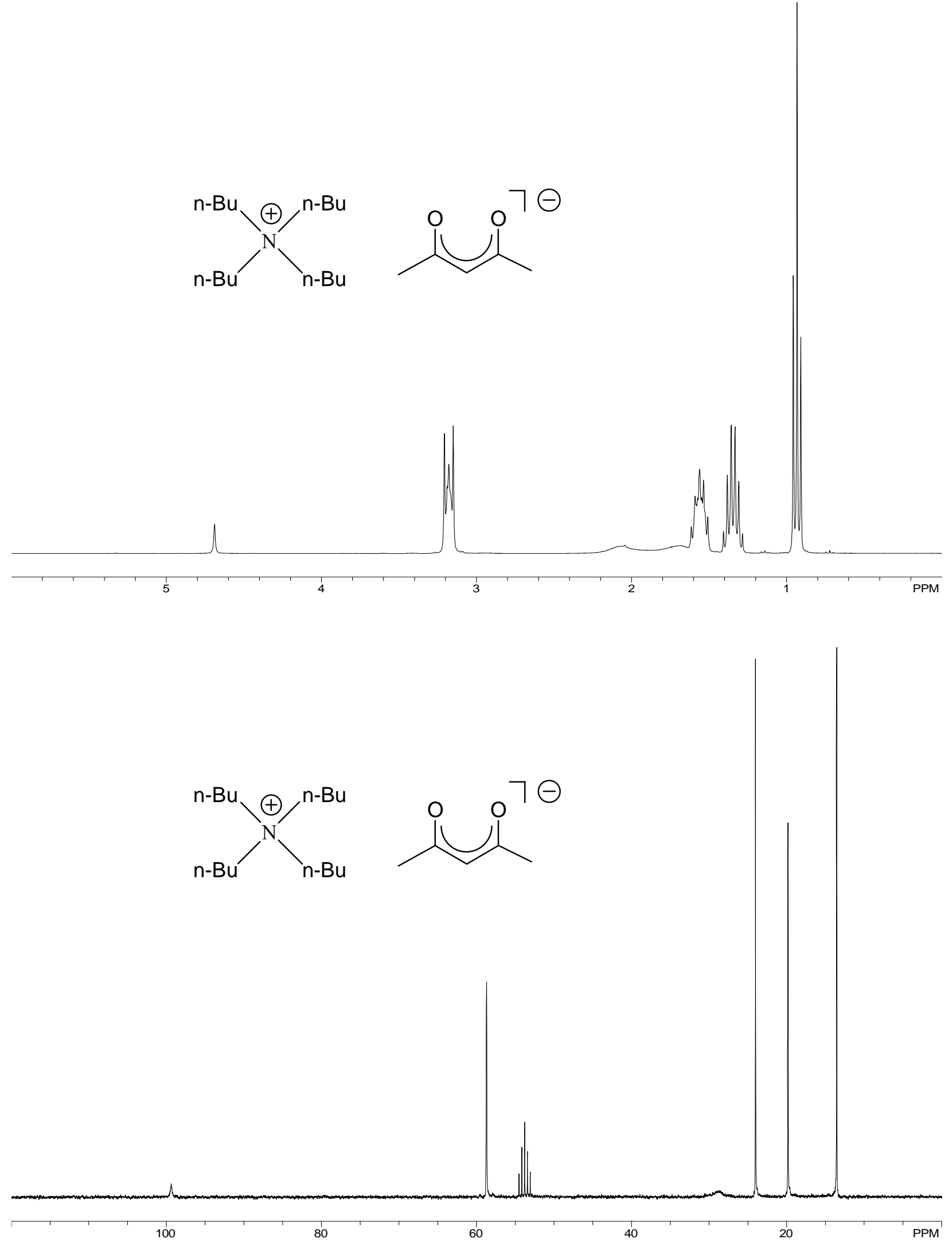
Figure 7. 1 equiv. of $[(S, S, R, R)$-Tangphos $] \mathrm{Rh}(\mathrm{acac})(33.8 \mathrm{mg})+1$ equiv. $(S, S, R, R)$-Tangphos (20 $\mathrm{mg}$ ) in $0.75 \mathrm{~mL}$ of THF-d8 $30 \mathrm{~min}$. (top spectrum) and $28 \mathrm{hr}$ (bottom spectrum) after mixing.
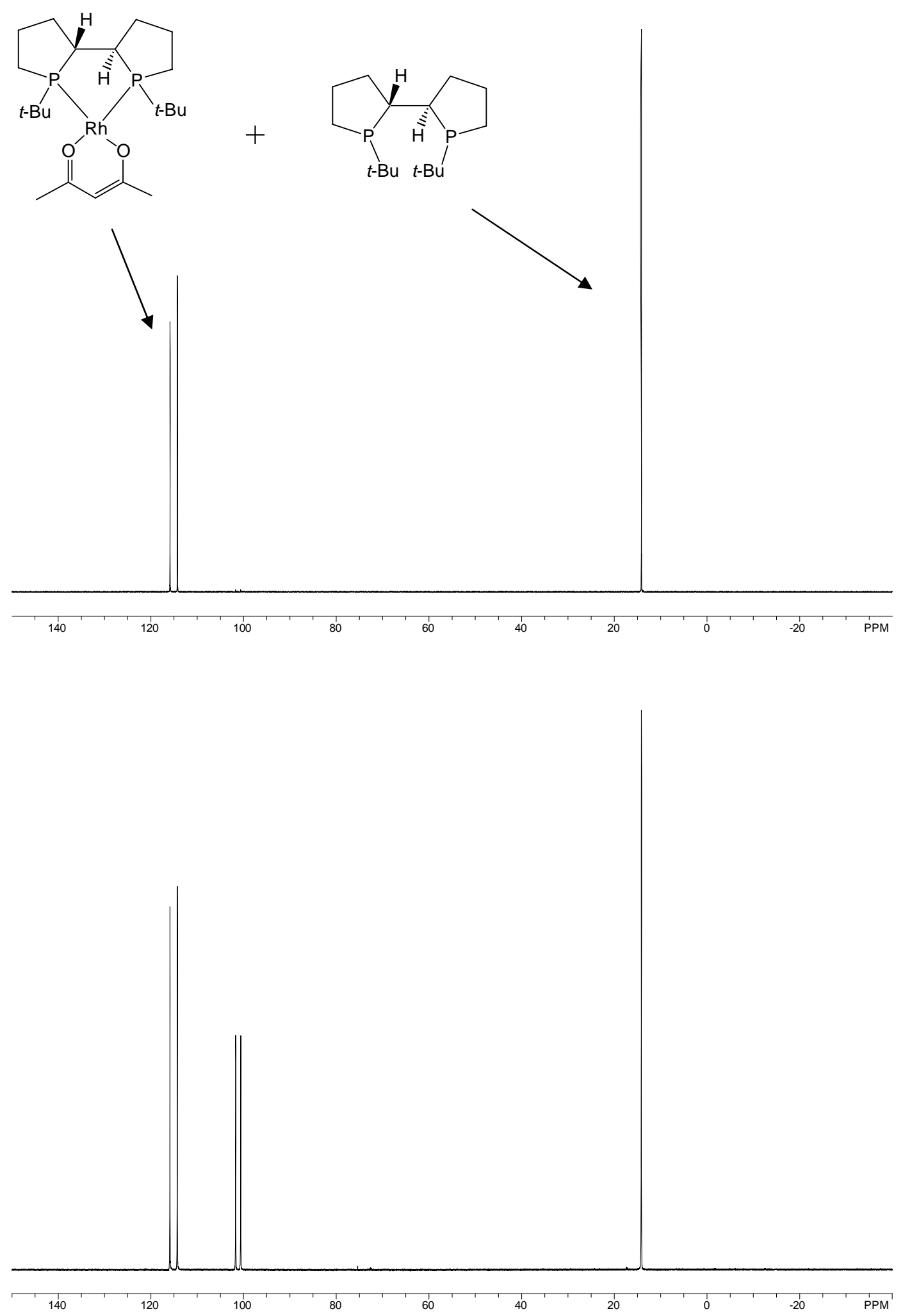
Figure $8 .{ }^{31} \mathrm{P}\left\{{ }^{1} \mathrm{H}\right\}$ spectra of reaction between $[(S, S, R, R)$-TangPhos) $) \mathrm{Rh}(\mathrm{acac})$ and $(S, S, R, R)$ TangPhos) taken after $1 \mathrm{~h}, 6 \mathrm{~h}$ and $21 \mathrm{~h}$ at $80{ }^{\circ} \mathrm{C}$ (in $0.5 \mathrm{~mL}$ of $\mathrm{C}_{6} \mathrm{D}_{6}$ and $0.25 \mathrm{~mL}$ of THF-d ${ }^{8}$ ).
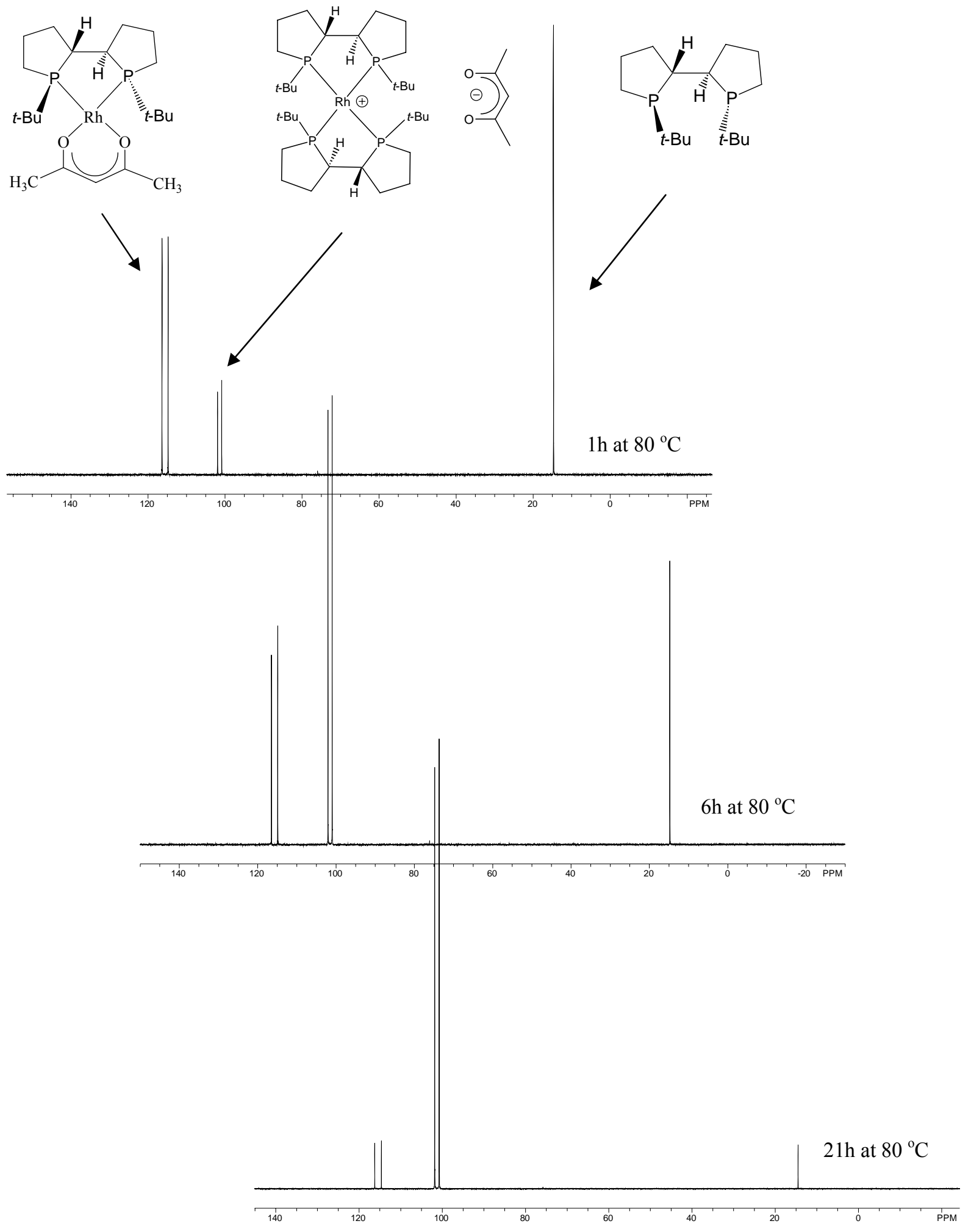
Figure 9. 1 equiv. of $\mathrm{Rh}(\mathrm{CO})_{2}$ (acac) $(25.6 \mathrm{mg})+1$ equiv. $(S, S, R, R)$-Tangphos $(28.4 \mathrm{mg})$ in 0.75 $\mathrm{mL}$ of THF-d 30 min. after mixing

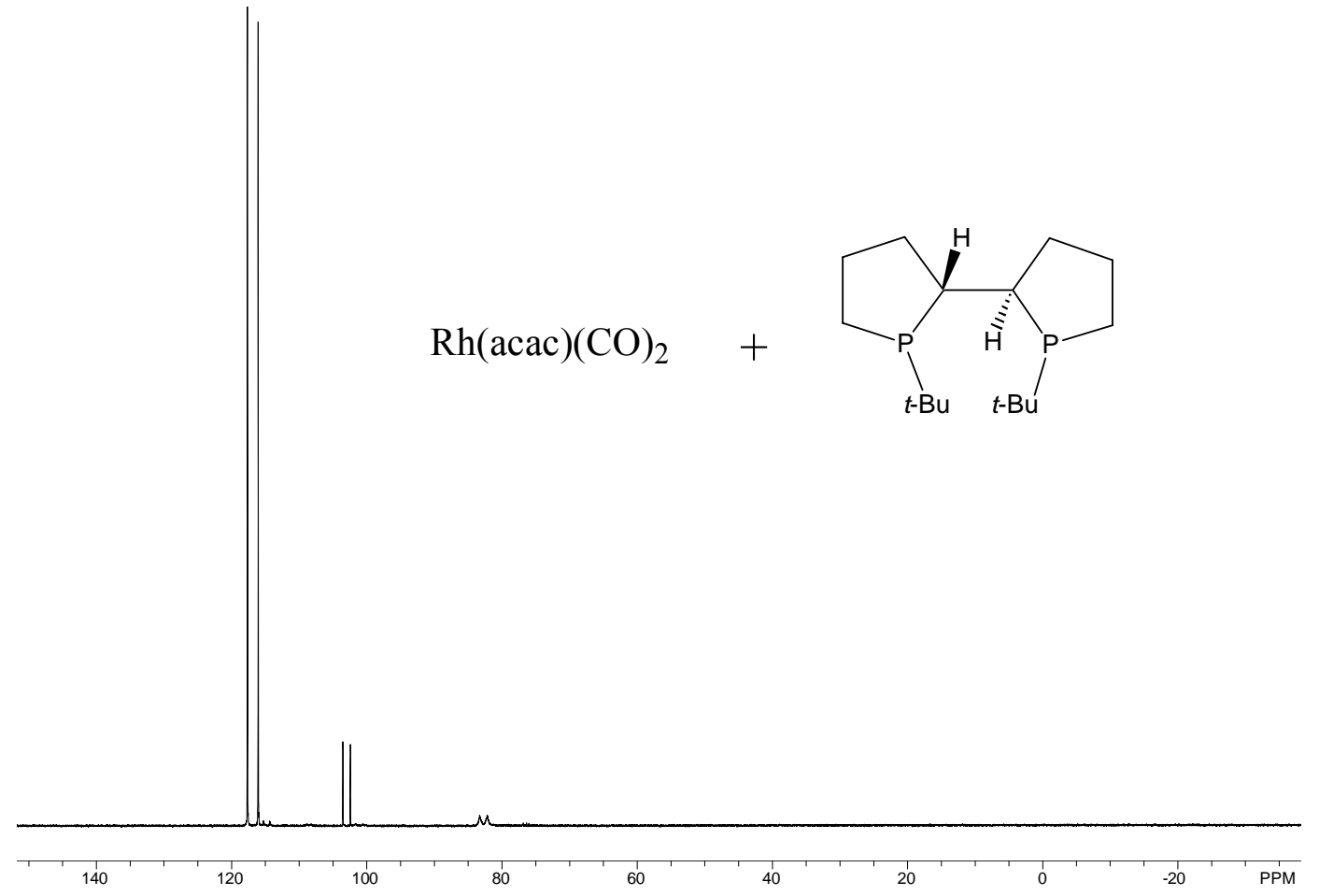

Figure 10. 1 equiv. of $\mathrm{Rh}(\mathrm{CO})_{2}$ (acac) $(25.9 \mathrm{mg})+2$ equiv. $(S, S, R, R)$-Tangphos $(57.5 \mathrm{mg})$ in 0.75 $\mathrm{mL}$ of THF-d 30 min. after mixing

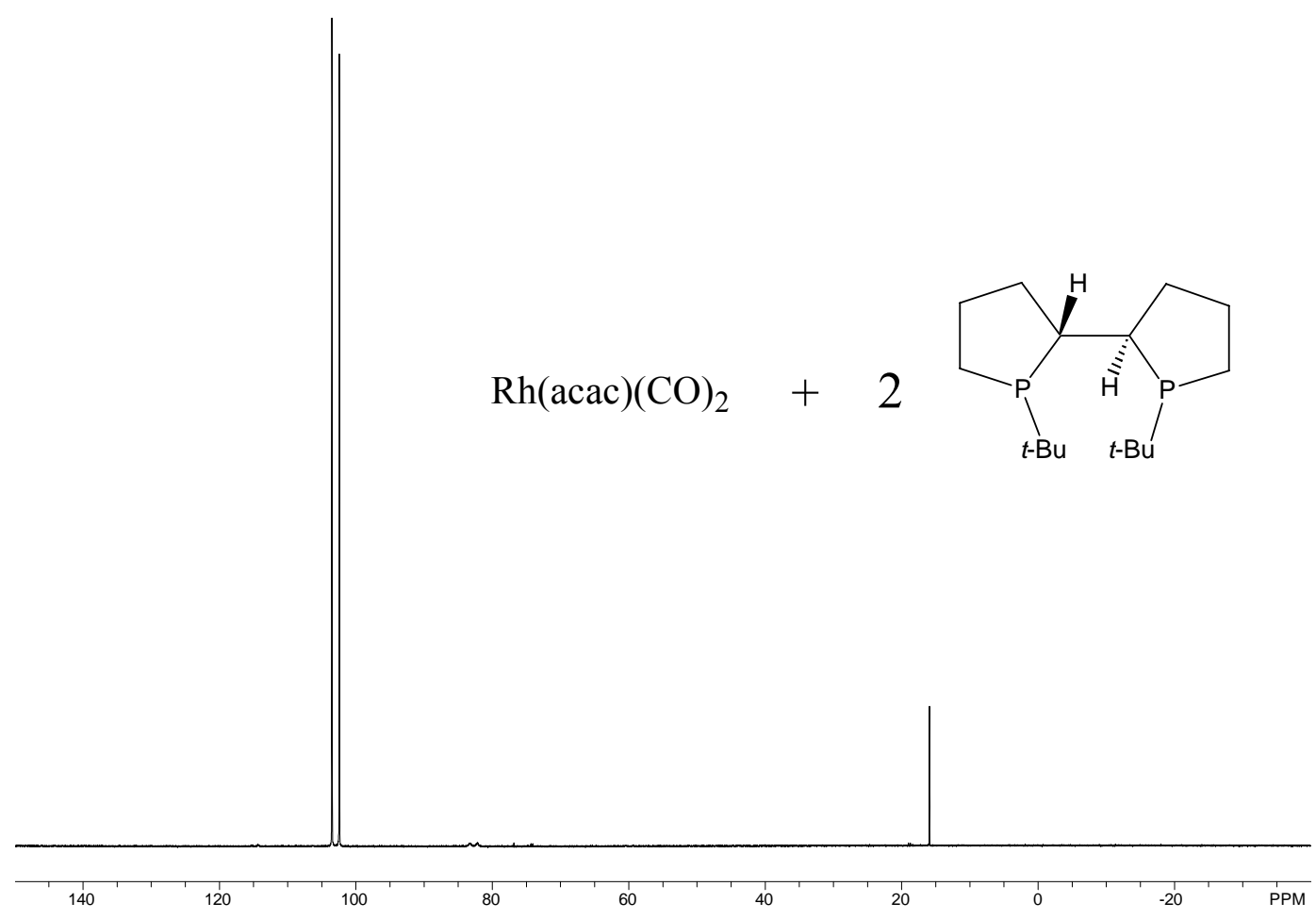


Table 1. Crystal data and structure refinement for $[(S, S, R, R)$-TangPhos $] \mathrm{Rh}(\mathrm{acac})(\mathbf{1 9})$.

Empirical formula

Formula weight

Temperature

Wavelength

Crystal system

Space group

Unit cell dimensions

Volume

Z

Density (calculated)

Absorption coefficient

$\mathrm{F}(000)$

Crystal size

Theta range for data collection

Index ranges

Reflections collected

Independent reflections

Completeness to theta $=27.50^{\circ}$

Absorption correction

Max. and min. transmission

Refinement method

Data / restraints / parameters

Goodness-of-fit on $\mathrm{F}^{2}$

Final R indices [I $>2 \operatorname{sigma}(\mathrm{I})]$

$\mathrm{R}$ indices (all data)

Absolute structure parameter

Largest diff. peak and hole
$\mathrm{C}_{21} \mathrm{H}_{39} \mathrm{O}_{2} \mathrm{P}_{2} \mathrm{Rh}$

488.37

173(2) K

$0.71073 \AA$

Trigonal

$\mathrm{P} 3{ }_{1} 21$

$\mathrm{a}=11.0103(4) \AA$

$\alpha=90^{\circ}$.

$\mathrm{b}=11.0103(4) \AA$

$\beta=90^{\circ}$.

$\mathrm{c}=33.178(3) \AA$

$\gamma=120^{\circ}$.

6

$1.397 \mathrm{Mg} / \mathrm{m}^{3}$

$0.886 \mathrm{~mm}^{-1}$

1536

$0.18 \times 0.17 \times 0.09 \mathrm{~mm}^{3}$

1.84 to $27.50^{\circ}$.

$-14 \leq \mathrm{h} \leq 13,-14 \leq \mathrm{k} \leq 11,-35 \leq \mathrm{l} \leq 43$

22592

$5320[\mathrm{R}(\mathrm{int})=0.0550]$

$99.9 \%$

Integration

0.9244 and 0.8636

Full-matrix least-squares on $\mathrm{F}^{2}$

$5320 / 0 / 243$

1.063

$\mathrm{R} 1=0.0270, \mathrm{wR} 2=0.0561[5023]$

$\mathrm{R} 1=0.0304, \mathrm{wR} 2=0.0570$

$0.00(2)$

0.304 and -0.375 e. $\AA^{-3}$

$\mathrm{R} 1=\sum\left(|| \mathrm{F}_{\mathrm{O}}|-| \mathrm{F}_{\mathrm{C}} \|\right) / \sum\left|\mathrm{F}_{\mathrm{O}}\right|$

$\mathrm{wR} 2=\left[\Sigma\left[\mathrm{w}\left(\mathrm{F}_{\mathrm{o}}{ }^{2}-\mathrm{F}_{\mathrm{c}}{ }^{2}\right)^{2}\right] / \Sigma\left[\mathrm{w}\left(\mathrm{F}_{\mathrm{o}}{ }^{2}\right)^{2}\right]\right]^{1 / 2}$

$\mathrm{S}=\left[\Sigma\left[\mathrm{w}\left(\mathrm{F}_{\mathrm{o}}^{2}-\mathrm{F}_{\mathrm{c}}{ }^{2}\right)^{2}\right] /(\mathrm{n}-\mathrm{p})\right]^{1 / 2}$

$\mathrm{w}=1 /\left[\sigma^{2}\left(\mathrm{~F}_{\mathrm{o}}{ }^{2}\right)+\left(\mathrm{m}^{*} \mathrm{p}\right)^{2}+\mathrm{n} * \mathrm{p}\right], \mathrm{p}=\left[\max \left(\mathrm{F}_{\mathrm{o}}^{2}, 0\right)+2^{*} \mathrm{~F}_{\mathrm{c}}^{2}\right] / 3, \mathrm{~m} \& \mathrm{n}$ are constants. 
Table 2. Atomic coordinates ( $\left.\times 10^{4}\right)$ and equivalent isotropic displacement parameters $\left(\AA^{2} \times 10^{3}\right)$ for $[(S, S, R, R)$ TangPhos] $\mathrm{Rh}$ (acac) (19). U(eq) is defined as one third of the trace of the orthogonalized $\mathrm{U}^{\mathrm{ij}}$ tensor.

\begin{tabular}{lrrrr}
\hline & $\mathrm{x}$ & $\mathrm{y}$ & $\mathrm{z}$ & $\mathrm{U}(\mathrm{eq})$ \\
\hline $\mathrm{Rh}$ & $6858(1)$ & $1986(1)$ & $9132(1)$ & $23(1)$ \\
$\mathrm{P} 1$ & $5996(1)$ & $-287(1)$ & $9181(1)$ & $24(1)$ \\
$\mathrm{P} 2$ & $4946(1)$ & $1620(1)$ & $9437(1)$ & $24(1)$ \\
O1 & $8642(2)$ & $2324(2)$ & $8817(1)$ & $29(1)$ \\
O2 & $7699(2)$ & $4170(2)$ & $9133(1)$ & $29(1)$ \\
C1 & $4270(3)$ & $-1217(3)$ & $9447(1)$ & $31(1)$ \\
C2 & $3210(3)$ & $-2175(3)$ & $9129(1)$ & $46(1)$ \\
C3 & $3926(3)$ & $-2696(3)$ & $8845(1)$ & $48(1)$ \\
C4 & $5340(3)$ & $-1429(3)$ & $8731(1)$ & $43(1)$ \\
C5 & $3888(3)$ & $-199(3)$ & $9646(1)$ & $32(1)$ \\
C6 & $4199(4)$ & $14(4)$ & $10100(1)$ & $50(1)$ \\
C7 & $4166(4)$ & $1313(4)$ & $10227(1)$ & $51(1)$ \\
C8 & $5104(3)$ & $2462(3)$ & $9932(1)$ & $41(1)$ \\
C9 & $7147(3)$ & $-874(3)$ & $9435(1)$ & $35(1)$ \\
C10 & $6464(3)$ & $-2467(3)$ & $9472(1)$ & $42(1)$ \\
C11 & $8487(3)$ & $-327(3)$ & $9190(1)$ & $57(1)$ \\
C12 & $7486(4)$ & $-232(4)$ & $9860(1)$ & $61(1)$ \\
C13 & $3755(3)$ & $2049(3)$ & $9143(1)$ & $28(1)$ \\
C14 & $3417(4)$ & $1279(4)$ & $8741(1)$ & $53(1)$ \\
C15 & $4538(4)$ & $3626(4)$ & $9062(1)$ & $52(1)$ \\
C16 & $2388(3)$ & $1636(4)$ & $9365(1)$ & $49(1)$ \\
C17 & $10780(3)$ & $3418(4)$ & $8467(1)$ & $44(1)$ \\
C18 & $9634(3)$ & $3493(3)$ & $8697(1)$ & $30(1)$ \\
C19 & $9771(3)$ & $4805(3)$ & $8756(1)$ & $30(1)$ \\
C20 & $8846(3)$ & $5075(3)$ & $8975(1)$ & $26(1)$ \\
C21 & $9247(3)$ & $6584(3)$ & $9040(1)$ & $34(1)$ \\
& & & & \\
\hline
\end{tabular}

Table 3. Bond lengths $[\AA]$ and angles $\left[^{\circ}\right]$ for $[(S, S, R, R)$-TangPhos $] \operatorname{Rh}($ acac) (19).

\begin{tabular}{ll|ll}
\hline Rh-O1 & $2.087(2)$ & C2-C3 & $1.512(5)$ \\
Rh-O2 & $2.101(2)$ & C2-H2A & 0.9900 \\
Rh-P2 & $2.1837(7)$ & C2-H2B & 0.9900 \\
Rh-P1 & $2.1945(7)$ & C3-C4 & $1.530(4)$ \\
P1-C4 & C3-H3A & 0.9900 \\
P1-C1 & C3-H3B & 0.9900 \\
P1-C9 & $1.849(3)$ & C4-H4A & 0.9900 \\
P2-C8 & $1.869(3)$ & C4-H4B & 0.9900 \\
P2-C5 & $1.885(3)$ & C5-C6 & $1.536(4)$ \\
P2-C13 & $1.852(3)$ & C5-H5A & 1.0000 \\
O1-C18 & C6-C7 & $1.509(5)$ \\
O2-C20 & C6-H6A & 0.9900 \\
C1-C5 & $1.875(3)$ & C6-H6B & 0.9900 \\
C1-C2 & $1.875(3)$ & C7-C8 & $1.523(5)$ \\
C1-H1A & $1.267(3)$ & C7-H7A & 0.9900
\end{tabular}




\begin{tabular}{|c|c|c|c|}
\hline C7-H7B & 0.9900 & C13-P2-Rh & $116.37(9)$ \\
\hline C8-H8A & 0.9900 & C18-O1-Rh & $126.58(18)$ \\
\hline C8-H8B & 0.9900 & $\mathrm{C} 20-\mathrm{O} 2-\mathrm{Rh}$ & $126.52(17)$ \\
\hline C9-C11 & $1.521(4)$ & $\mathrm{C} 5-\mathrm{C} 1-\mathrm{C} 2$ & $114.1(2)$ \\
\hline C9-C10 & $1.529(4)$ & C5-C1-P1 & $112.28(19)$ \\
\hline C9-C12 & $1.535(5)$ & C2-C1-P1 & $105.5(2)$ \\
\hline C10-H10A & 0.9800 & C5-C1-H1A & 108.3 \\
\hline C10-H10B & 0.9800 & C2-C1-H1A & 108.3 \\
\hline $\mathrm{C} 10-\mathrm{H} 10 \mathrm{C}$ & 0.9800 & P1-C1-H1A & 108.3 \\
\hline C11-H11A & 0.9800 & C3-C2-C1 & 109.1(2) \\
\hline C11-H11B & 0.9800 & $\mathrm{C} 3-\mathrm{C} 2-\mathrm{H} 2 \mathrm{~A}$ & 109.9 \\
\hline C11-H11C & 0.9800 & $\mathrm{C} 1-\mathrm{C} 2-\mathrm{H} 2 \mathrm{~A}$ & 109.9 \\
\hline C12-H12A & 0.9800 & C3-C2-H2B & 109.9 \\
\hline C12-H12B & 0.9800 & $\mathrm{C} 1-\mathrm{C} 2-\mathrm{H} 2 \mathrm{~B}$ & 109.9 \\
\hline $\mathrm{C} 12-\mathrm{H} 12 \mathrm{C}$ & 0.9800 & $\mathrm{H} 2 \mathrm{~A}-\mathrm{C} 2-\mathrm{H} 2 \mathrm{~B}$ & 108.3 \\
\hline C13-C14 & $1.524(4)$ & C2-C3-C4 & 107.1(3) \\
\hline C13-C16 & $1.526(4)$ & $\mathrm{C} 2-\mathrm{C} 3-\mathrm{H} 3 \mathrm{~A}$ & 110.3 \\
\hline C13-C15 & $1.528(4)$ & C4-C3-H3A & 110.3 \\
\hline C14-H14A & 0.9800 & C2-C3-H3B & 110.3 \\
\hline C14-H14B & 0.9800 & C4-C3-H3B & 110.3 \\
\hline C14-H14C & 0.9800 & $\mathrm{H} 3 \mathrm{~A}-\mathrm{C} 3-\mathrm{H} 3 \mathrm{~B}$ & 108.6 \\
\hline C15-H15A & 0.9800 & C3-C4-P1 & 107.1(2) \\
\hline C15-H15B & 0.9800 & C3-C4-H4A & 110.3 \\
\hline C15-H15C & 0.9800 & P1-C4-H4A & 110.3 \\
\hline C16-H16A & 0.9800 & C3-C4-H4B & 110.3 \\
\hline C16-H16B & 0.9800 & P1-C4-H4B & 110.3 \\
\hline $\mathrm{C} 16-\mathrm{H} 16 \mathrm{C}$ & 0.9800 & H4A-C4-H4B & 108.5 \\
\hline C17-C18 & $1.512(4)$ & C1-C5-C6 & $114.4(2)$ \\
\hline C17-H17A & 0.9800 & C1-C5-P2 & $111.80(18)$ \\
\hline C17-H17B & 0.9800 & C6-C5-P2 & $103.2(2)$ \\
\hline $\mathrm{C} 17-\mathrm{H} 17 \mathrm{C}$ & 0.9800 & C1-C5-H5A & 109.1 \\
\hline C18-C19 & $1.389(4)$ & C6-C5-H5A & 109.1 \\
\hline C19-C20 & $1.399(4)$ & P2-C5-H5A & 109.1 \\
\hline C19-H19A & 0.9500 & C7-C6-C5 & 108.1(3) \\
\hline C20-C21 & $1.505(4)$ & C7-C6-H6A & 110.1 \\
\hline $\mathrm{C} 21-\mathrm{H} 21 \mathrm{~A}$ & 0.9800 & C5-C6-H6A & 110.1 \\
\hline C21-H21B & 0.9800 & C7-C6-H6B & 110.1 \\
\hline \multirow[t]{2}{*}{$\mathrm{C} 21-\mathrm{H} 21 \mathrm{C}$} & 0.9800 & C5-C6-H6B & 110.1 \\
\hline & & H6A-C6-H6B & 108.4 \\
\hline $\mathrm{O} 1-\mathrm{Rh}-\mathrm{O} 2$ & $87.70(7)$ & C6-C7-C8 & $106.2(3)$ \\
\hline O1-Rh-P2 & $177.54(6)$ & C6-C7-H7A & 110.5 \\
\hline O2-Rh-P2 & $92.46(5)$ & C8-C7-H7A & 110.5 \\
\hline O1-Rh-P1 & $94.13(6)$ & C6-C7-H7B & 110.5 \\
\hline O2-Rh-P1 & $175.64(5)$ & C8-C7-H7B & 110.5 \\
\hline P2-Rh-P1 & $85.88(3)$ & H7A-C7-H7B & 108.7 \\
\hline C4-P1-C1 & $93.65(14)$ & C7-C8-P2 & 107.4(2) \\
\hline C4-P1-C9 & $103.27(15)$ & C7-C8-H8A & 110.2 \\
\hline C1-P1-C9 & $105.94(12)$ & P2-C8-H8A & 110.2 \\
\hline C4-P1-Rh & $120.99(11)$ & C7-C8-H8B & 110.2 \\
\hline C1-P1-Rh & 113.62(9) & P2-C8-H8B & 110.2 \\
\hline C9-P1-Rh & $116.27(10)$ & H8A-C $8-H 8 B$ & 108.5 \\
\hline C8-P2-C5 & $93.38(14)$ & C11-C9-C10 & $108.6(2)$ \\
\hline C8-P2-C13 & $103.75(13)$ & $\mathrm{C} 11-\mathrm{C} 9-\mathrm{C} 12$ & $110.0(3)$ \\
\hline C5-P2-C13 & $108.83(13)$ & $\mathrm{C} 10-\mathrm{C} 9-\mathrm{C} 12$ & $108.8(3)$ \\
\hline C8-P2-Rh & $118.53(10)$ & C11-C9-P1 & $108.1(2)$ \\
\hline $\mathrm{C} 5-\mathrm{P} 2-\mathrm{Rh}$ & $113.28(9)$ & C10-C9-P1 & $113.5(2)$ \\
\hline
\end{tabular}




\begin{tabular}{ll} 
C12-C9-P1 & $107.7(2)$ \\
C9-C10-H10A & 109.5 \\
C9-C10-H10B & 109.5 \\
H10A-C10-H10B & 109.5 \\
C9-C10-H10C & 109.5 \\
H10A-C10-H10C & 109.5 \\
H10B-C10-H10C & 109.5 \\
C9-C11-H11A & 109.5 \\
C9-C11-H11B & 109.5 \\
H11A-C11-H11B & 109.5 \\
C9-C11-H11C & 109.5 \\
H11A-C11-H11C & 109.5 \\
H11B-C11-H11C & 109.5 \\
C9-C12-H12A & 109.5 \\
C9-C12-H12B & 109.5 \\
H12A-C12-H12B & 109.5 \\
C9-C12-H12C & 109.5 \\
H12A-C12-H12C & 109.5 \\
H12B-C12-H12C & 109.5 \\
C14-C13-C16 & $109.1(3)$ \\
C14-C13-C15 & $108.7(3)$ \\
C16-C13-C15 & $109.7(3)$ \\
C14-C13-P2 & $108.75(19)$ \\
C16-C13-P2 & $112.55(19)$ \\
C15-C13-P2 & $108.05(19)$ \\
C13-C14-H14A & 109.5 \\
C13-C14-H14B & 109.5 \\
H14A-C14-H14B & 109.5 \\
C13-C14-H14C & 109.5 \\
H14A-C14-H14C & 109.5 \\
H14B-C14-H14C & 109.5 \\
C13-C15-H15A & 109.5 \\
C13-C15-H15B & 109.5 \\
H15A-C15-H15B & 109.5 \\
C13-C15-H15C & 109.5 \\
H15A-C15-H15C & 109.5 \\
H15B-C15-H15C & 109.5 \\
C13-C16-H16A & 109.5 \\
C13-C16-H16B & 109.5 \\
H16A-C16-H16B & 109.5 \\
C13-C16-H16C & 109.5 \\
H16A-C16-H16C & 109.5 \\
H16B-C16-H16C & 109.5 \\
C18-C17-H17A & 109.5 \\
C18-C17-H17B & 109.5 \\
H17A-C17-H17B & 109.5 \\
C18-C17-H17C & 109.5 \\
H17A-C17-H17C & 109.5 \\
H17B-C17-H17C & 109.5 \\
O1-C18-C19 & $127.0(2)$ \\
O1-C18-C17 & $115.0(3)$ \\
C19-C18-C17 & $118.0(3)$ \\
C18-C19-C20 & $117.6(3)$ \\
C18-C19-H19A & 117.2 \\
C20-C19-H19A & $126.4(3)$ \\
O2-C20-C19 & \\
\hline & \\
&
\end{tabular}

$\begin{array}{ll}\text { O2-C20-C21 } & 115.8(2) \\ \text { C19-C20-C21 } & 117.7(2) \\ \text { C20-C21-H21A } & 109.5 \\ \text { C20-C21-H21B } & 109.5 \\ \text { H21A-C21-H21B } & 109.5 \\ \text { C20-C21-H21C } & 109.5 \\ \text { H21A-C21-H21C } & 109.5 \\ \text { H21B-C21-H21C } & 109.5\end{array}$


Table 4. Anisotropic displacement parameters $\left(\AA^{2} \times 10^{3}\right)$ for $[(S, S, R, R)$-TangPhos $] \mathrm{Rh}($ acac $)(19)$. The anisotropic

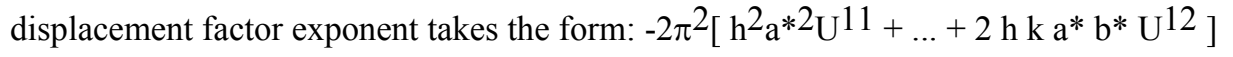

\begin{tabular}{lcccccc}
\hline & $\mathrm{U}^{11}$ & $\mathrm{U}^{22}$ & $\mathrm{U}^{33}$ & $\mathrm{U}^{23}$ & $\mathrm{U}^{13}$ & $\mathrm{U}^{12}$ \\
\hline $\mathrm{Rh}$ & $19(1)$ & $23(1)$ & $27(1)$ & $2(1)$ & $2(1)$ & $9(1)$ \\
$\mathrm{P} 1$ & $22(1)$ & $24(1)$ & $26(1)$ & $1(1)$ & $1(1)$ & $10(1)$ \\
$\mathrm{P} 2$ & $21(1)$ & $27(1)$ & $24(1)$ & $0(1)$ & $0(1)$ & $12(1)$ \\
$\mathrm{O} 1$ & $24(1)$ & $28(1)$ & $33(1)$ & $1(1)$ & $6(1)$ & $11(1)$ \\
$\mathrm{O} 2$ & $25(1)$ & $25(1)$ & $36(1)$ & $1(1)$ & $1(1)$ & $13(1)$ \\
$\mathrm{C} 1$ & $22(1)$ & $25(1)$ & $40(2)$ & $9(1)$ & $4(1)$ & $8(1)$ \\
$\mathrm{C} 2$ & $28(2)$ & $32(2)$ & $71(2)$ & $-3(2)$ & $-8(2)$ & $9(1)$ \\
$\mathrm{C} 3$ & $48(2)$ & $32(2)$ & $54(2)$ & $-11(2)$ & $-18(2)$ & $12(2)$ \\
$\mathrm{C} 4$ & $52(2)$ & $37(2)$ & $33(2)$ & $-5(1)$ & $-4(1)$ & $18(2)$ \\
$\mathrm{C} 5$ & $23(1)$ & $35(2)$ & $38(2)$ & $12(1)$ & $8(1)$ & $14(1)$ \\
$\mathrm{C} 6$ & $62(2)$ & $72(2)$ & $36(2)$ & $18(2)$ & $15(2)$ & $48(2)$ \\
$\mathrm{C} 7$ & $60(2)$ & $82(2)$ & $24(2)$ & $1(2)$ & $4(2)$ & $46(2)$ \\
$\mathrm{C} 8$ & $38(2)$ & $54(2)$ & $34(2)$ & $-14(1)$ & $-7(1)$ & $26(2)$ \\
$\mathrm{C} 9$ & $28(1)$ & $32(2)$ & $46(2)$ & $4(1)$ & $0(1)$ & $18(1)$ \\
$\mathrm{C} 10$ & $37(2)$ & $35(2)$ & $57(2)$ & $11(2)$ & $8(2)$ & $21(1)$ \\
$\mathrm{C} 11$ & $33(2)$ & $40(2)$ & $104(3)$ & $16(2)$ & $12(2)$ & $23(2)$ \\
$\mathrm{C} 12$ & $75(3)$ & $66(2)$ & $66(2)$ & $-19(2)$ & $-38(2)$ & $53(2)$ \\
$\mathrm{C} 13$ & $28(1)$ & $32(2)$ & $26(1)$ & $4(1)$ & $2(1)$ & $18(1)$ \\
$\mathrm{C} 14$ & $71(2)$ & $74(3)$ & $37(2)$ & $-11(2)$ & $-22(2)$ & $54(2)$ \\
$\mathrm{C} 15$ & $42(2)$ & $41(2)$ & $77(2)$ & $14(2)$ & $-4(2)$ & $24(2)$ \\
$\mathrm{C} 16$ & $34(2)$ & $67(2)$ & $56(2)$ & $14(2)$ & $4(2)$ & $33(2)$ \\
$\mathrm{C} 17$ & $34(2)$ & $46(2)$ & $49(2)$ & $1(2)$ & $14(1)$ & $17(2)$ \\
$\mathrm{C} 18$ & $23(1)$ & $37(2)$ & $25(1)$ & $1(1)$ & $-1(1)$ & $12(1)$ \\
$\mathrm{C} 19$ & $23(1)$ & $30(2)$ & $30(2)$ & $3(1)$ & $4(1)$ & $8(1)$ \\
$\mathrm{C} 20$ & $24(1)$ & $26(1)$ & $26(1)$ & $2(1)$ & $-6(1)$ & $12(1)$ \\
$\mathrm{C} 21$ & $32(2)$ & $26(1)$ & $40(2)$ & $3(1)$ & $-2(1)$ & $13(1)$ \\
& & & & & & \\
\hline
\end{tabular}

Table 5. Hydrogen coordinates $\left(\times 10^{4}\right)$ and isotropic displacement parameters $\left(\AA^{2} \times 10^{3}\right)$ for $[(S, S, R, R)$ TangPhos]Rh(acac) (19).

\begin{tabular}{lrrrr}
\hline & $\mathrm{x}$ & $\mathrm{y}$ & $\mathrm{z}$ & $\mathrm{U}(\mathrm{eq})$ \\
\hline H1A & & & & \\
H2A & 4330 & -1822 & 9662 & 37 \\
H2B & 2406 & -2981 & 9262 & 56 \\
H3A & 2850 & -1649 & 8975 & 56 \\
H3B & 3347 & -3113 & 8601 & 57 \\
H4A & 4068 & -3419 & 8979 & 57 \\
H4B & 5224 & -916 & 8503 & 52 \\
H5A & 6009 & -1737 & 8650 & 52 \\
H6A & 2870 & -540 & 9601 & 38 \\
& 3487 & -810 & 10251 & 60
\end{tabular}




\begin{tabular}{lrrrl} 
H6B & 5133 & 128 & 10157 & 60 \\
H7A & 4522 & 1581 & 10506 & 61 \\
H7B & 3196 & 1148 & 10216 & 61 \\
H8A & 6090 & 2936 & 10026 & 49 \\
H8B & 4804 & 3171 & 9911 & 49 \\
H10A & 6230 & -2888 & 9203 & 63 \\
H10B & 5607 & -2831 & 9633 & 63 \\
H10C & 7118 & -2702 & 9603 & 63 \\
H11A & 9089 & -647 & 9312 & 85 \\
H11B & 8983 & 700 & 9187 & 85 \\
H11C & 8254 & -682 & 8914 & 85 \\
H12A & 8186 & -413 & 9986 & 92 \\
H12B & 6631 & -655 & 10023 & 92 \\
H12C & 7860 & 782 & 9841 & 92 \\
H14A & 2811 & 270 & 8787 & 79 \\
H14B & 4289 & 1451 & 8611 & 79 \\
H14C & 2934 & 1619 & 8566 & 79 \\
H15A & 3963 & 3864 & 8890 & 78 \\
H15B & 5427 & 3892 & 8925 & 78 \\
H15C & 4728 & 4134 & 9317 & 74 \\
H16A & 2600 & 2064 & 9633 & 74 \\
H16B & 613 & 9391 & 74 \\
H16C & 1842 & 1964 & 9212 & 66 \\
H17A & 1845 & 3573 & 8652 & 66 \\
H17B & 11537 & 4140 & 8257 & 66 \\
H17C & 11149 & 2490 & 8342 & 36 \\
H19A & 10396 & 5587 & 8638 & 51 \\
H21A & 10562 & 6641 & 9094 & 51 \\
H21B & 8402 & 7128 & 8797 & \\
H21C & 9712 & 6964 & 9270 & \\
& 9888 & & & \\
\hline
\end{tabular}


Table 6. Crystal data and structure refinement for $\left.\left[((S, S, R, R)-\text { TangPhos })_{2}\right] \mathrm{Rh}\right][\mathrm{acac}]$.

Identification code

Empirical formula

Formula weight

Temperature

Wavelength

Crystal system

Space group

Unit cell dimensions

Volume

Z

Density (calculated)

Absorption coefficient

$\mathrm{F}(000)$

Crystal size

Theta range for data collection

Index ranges

Reflections collected

Independent reflections

Completeness to theta $=27.50^{\circ}$

Absorption correction

Max. and min. transmission

Refinement method

Data / restraints / parameters

Goodness-of-fit on $\mathrm{F}^{2}$

Final R indices [I $>2$ sigma(I)]

$\mathrm{R}$ indices (all data)

Absolute structure parameter

Largest diff. peak and hole jkx27

$\mathrm{C}_{37} \mathrm{H}_{71} \mathrm{O}_{2} \mathrm{P}_{4} \mathrm{Rh}$

774.73

173(2) K

$0.71073 \AA$

Orthorhombic

$\mathrm{P} 2{ }_{1} 2_{1} 2_{1}$

$\mathrm{a}=12.960(1) \AA$ $\alpha=90^{\circ}$.

$\mathrm{b}=14.8220(11) \AA$

$\beta=90^{\circ}$.

$\mathrm{c}=20.9965(16) \AA$

$\gamma=90^{\circ}$.
4033.3(5) $\AA^{3}$

4

$1.276 \mathrm{Mg} / \mathrm{m}^{3}$

$0.611 \mathrm{~mm}^{-1}$

1656

$0.14 \times 0.12 \times 0.09 \mathrm{~mm}^{3}$

1.68 to $27.50^{\circ}$.

$-11 \leq \mathrm{h} \leq 16,-12 \leq \mathrm{k} \leq 18,-26 \leq \mathrm{l} \leq 22$

15163

$8785[\mathrm{R}(\mathrm{int})=0.0751]$

$97.0 \%$

Integration

0.9523 and 0.9145

Full-matrix least-squares on $\mathrm{F}^{2}$

8785 / 0 / 397

1.000

$\mathrm{R} 1=0.0567, \mathrm{wR} 2=0.1241[6624]$

$\mathrm{R} 1=0.0839, \mathrm{wR} 2=0.1357$

$-0.04(4)$

0.722 and -1.001 e. $\AA^{-3}$

$\mathrm{R} 1=\sum\left(|| \mathrm{F}_{\mathrm{O}}|-| \mathrm{F}_{\mathrm{C}} \mid\right) / \sum\left|\mathrm{F}_{\mathrm{O}}\right|$

$\mathrm{wR} 2=\left[\Sigma\left[\mathrm{w}\left(\mathrm{F}_{\mathrm{o}}{ }^{2}-\mathrm{F}_{\mathrm{c}}{ }^{2}\right)^{2}\right] / \Sigma\left[\mathrm{w}\left(\mathrm{F}_{\mathrm{o}}^{2}\right)^{2}\right]\right]^{1 / 2}$

$\mathrm{S}=\left[\Sigma\left[\mathrm{w}\left(\mathrm{F}_{\mathrm{O}}^{2}-\mathrm{F}_{\mathrm{c}}^{2}\right)^{2}\right] /(\mathrm{n}-\mathrm{p})\right]^{1 / 2}$

$\mathrm{w}=1 /\left[\sigma^{2}\left(\mathrm{~F}_{\mathrm{O}}{ }^{2}\right)+(\mathrm{m} * \mathrm{p})^{2}+\mathrm{n} * \mathrm{p}\right], \mathrm{p}=\left[\max \left(\mathrm{F}_{\mathrm{O}}{ }^{2}, 0\right)+2^{*} \mathrm{~F}_{\mathrm{c}}{ }^{2}\right] / 3, \mathrm{~m} \& \mathrm{n}$ are constants. 
Table 7. Atomic coordinates ( $\left.\times 10^{4}\right)$ and equivalent isotropic displacement parameters $\left(\AA^{2} \times 10^{3}\right)$ for $[((S, S, R, R)$ TangPhos $\left.\left.)_{2}\right] \mathrm{Rh}\right][\mathrm{acac}]$. U(eq) is defined as one third of the trace of the orthogonalized $\mathrm{U}^{\mathrm{ij}}$ tensor.

\begin{tabular}{|c|c|c|c|c|}
\hline & $\mathrm{x}$ & $\mathrm{y}$ & $\mathrm{z}$ & $\mathrm{U}(\mathrm{eq})$ \\
\hline $\mathrm{Rh}$ & $3187(1)$ & $4965(1)$ & 1418(1) & $20(1)$ \\
\hline P1 & $4854(1)$ & $5429(1)$ & 1551(1) & $23(1)$ \\
\hline P2 & $3960(1)$ & $3831(1)$ & $797(1)$ & $23(1)$ \\
\hline P3 & $1582(1)$ & $4387(1)$ & $1748(1)$ & $25(1)$ \\
\hline P4 & $2420(1)$ & $6329(1)$ & 1589(1) & $24(1)$ \\
\hline $\mathrm{C} 1$ & $5755(4)$ & 4932(5) & $956(3)$ & $29(1)$ \\
\hline $\mathrm{C} 2$ & $5961(6)$ & $5716(5)$ & $505(3)$ & $42(2)$ \\
\hline $\mathrm{C} 3$ & $6196(6)$ & $6531(4)$ & $920(3)$ & $41(2)$ \\
\hline $\mathrm{C} 4$ & $5318(4)$ & $6599(4)$ & $1422(3)$ & $32(1)$ \\
\hline $\mathrm{C} 5$ & $5370(4)$ & 4057(4) & $648(3)$ & $26(1)$ \\
\hline C6 & $5943(5)$ & $3198(4)$ & $871(4)$ & $37(2)$ \\
\hline C7 & $5246(5)$ & $2383(4)$ & $784(3)$ & $34(1)$ \\
\hline C8 & $4177(5)$ & $2648(4)$ & 1042(3) & $32(1)$ \\
\hline C9 & $5363(4)$ & $5126(5)$ & 2362(2) & $29(1)$ \\
\hline $\mathrm{C} 10$ & $6541(5)$ & $5298(5)$ & $2416(3)$ & $49(2)$ \\
\hline $\mathrm{C} 11$ & $4811(5)$ & $5707(4)$ & $2868(3)$ & $38(2)$ \\
\hline $\mathrm{C} 12$ & $5167(5)$ & 4134(4) & $2500(3)$ & $35(2)$ \\
\hline $\mathrm{C} 13$ & $3405(4)$ & $3779(4)$ & $-26(3)$ & $31(1)$ \\
\hline C14 & $3635(5)$ & $4663(4)$ & $-367(3)$ & $38(2)$ \\
\hline C15 & $2237(5)$ & $3651(5)$ & $36(3)$ & $38(2)$ \\
\hline C16 & $3831(6)$ & $3001(5)$ & $-430(3)$ & $42(2)$ \\
\hline $\mathrm{C} 21$ & $722(4)$ & $5304(4)$ & $2076(3)$ & $28(1)$ \\
\hline $\mathrm{C} 22$ & $-297(4)$ & $5236(4)$ & $1688(3)$ & $36(2)$ \\
\hline $\mathrm{C} 23$ & $-492(5)$ & $4268(5)$ & $1509(4)$ & $43(2)$ \\
\hline $\mathrm{C} 24$ & $535(4)$ & $3883(4)$ & $1257(3)$ & $32(1)$ \\
\hline $\mathrm{C} 25$ & $1222(4)$ & $6244(4)$ & 2074(3) & $30(1)$ \\
\hline $\mathrm{C} 26$ & $1555(5)$ & $6595(4)$ & $2725(3)$ & $33(2)$ \\
\hline $\mathrm{C} 27$ & 2161(5) & 7464(4) & 2595(3) & $34(2)$ \\
\hline $\mathrm{C} 28$ & 2973(5) & $7252(4)$ & 2079(3) & $31(1)$ \\
\hline C29 & $1706(5)$ & $3597(4)$ & 2443(3) & $31(1)$ \\
\hline $\mathrm{C} 30$ & $669(5)$ & $3226(5)$ & $2682(4)$ & $44(2)$ \\
\hline C31 & $2376(5)$ & 2790(4) & 2233(3) & $38(2)$ \\
\hline $\mathrm{C} 32$ & $2238(5)$ & 4095(4) & 2996(3) & $38(2)$ \\
\hline C33 & 2051(4) & $6886(4)$ & $815(3)$ & $29(1)$ \\
\hline C34 & 1484(5) & $6222(4)$ & $400(3)$ & $34(2)$ \\
\hline C35 & $3038(5)$ & $7200(4)$ & $481(3)$ & $36(2)$ \\
\hline C36 & $1368(5)$ & 7721(4) & 921(3) & $41(2)$ \\
\hline $\mathrm{O} 1$ & $6352(4)$ & $3329(3)$ & $-826(3)$ & $61(2)$ \\
\hline $\mathrm{O} 2$ & $8123(4)$ & $5717(3)$ & $-1190(2)$ & $42(1)$ \\
\hline C41 & $7638(5)$ & $4382(4)$ & $-643(3)$ & $42(2)$ \\
\hline $\mathrm{C} 42$ & $6766(6)$ & 4072(4) & $-961(3)$ & $38(2)$ \\
\hline C43 & $6257(5)$ & $4622(5)$ & $-1476(3)$ & $48(2)$ \\
\hline C44 & $8242(5)$ & $5146(4)$ & $-763(3)$ & $36(1)$ \\
\hline $\mathrm{C} 45$ & $9159(6)$ & $5297(6)$ & $-318(4)$ & $66(3)$ \\
\hline
\end{tabular}


Table 8. Bond lengths $[\AA]$ and angles $\left[{ }^{\circ}\right]$ for $\left.\left[((S, S, R, R) \text {-TangPhos })_{2}\right] \mathrm{Rh}\right][\mathrm{acac}]$.

\begin{tabular}{|c|c|c|c|}
\hline Rh-P4 & $2.281(2)$ & C14-H14C & 0.9800 \\
\hline Rh-P1 & $2.284(1)$ & C15-H15A & 0.9800 \\
\hline Rh-P2 & $2.351(2)$ & C15-H15B & 0.9800 \\
\hline Rh-P3 & $2.355(1)$ & $\mathrm{C} 15-\mathrm{H} 15 \mathrm{C}$ & 0.9800 \\
\hline P1-C4 & $1.855(6)$ & C16-H16A & 0.9800 \\
\hline $\mathrm{P} 1-\mathrm{C} 1$ & $1.861(5)$ & C16-H16B & 0.9800 \\
\hline P1-C9 & $1.880(5)$ & $\mathrm{C} 16-\mathrm{H} 16 \mathrm{C}$ & 0.9800 \\
\hline P2-C8 & $1.849(6)$ & C21-C25 & $1.536(8)$ \\
\hline P2-C13 & $1.873(6)$ & C21-C22 & $1.554(8)$ \\
\hline P2-C5 & $1.883(6)$ & $\mathrm{C} 21-\mathrm{H} 21 \mathrm{~A}$ & 1.0000 \\
\hline P3-C24 & $1.860(6)$ & C22-C23 & $1.505(9)$ \\
\hline P3-C29 & $1.877(6)$ & $\mathrm{C} 22-\mathrm{H} 22 \mathrm{~A}$ & 0.9900 \\
\hline P3-C21 & $1.888(6)$ & $\mathrm{C} 22-\mathrm{H} 22 \mathrm{~B}$ & 0.9900 \\
\hline P4-C28 & $1.856(6)$ & C23-C24 & $1.542(9)$ \\
\hline P4-C25 & $1.861(6)$ & $\mathrm{C} 23-\mathrm{H} 23 \mathrm{~A}$ & 0.9900 \\
\hline P4-C33 & $1.886(6)$ & C23-H23B & 0.9900 \\
\hline $\mathrm{C} 1-\mathrm{C} 2$ & $1.522(9)$ & $\mathrm{C} 24-\mathrm{H} 24 \mathrm{~A}$ & 0.9900 \\
\hline C1-C5 & $1.534(8)$ & $\mathrm{C} 24-\mathrm{H} 24 \mathrm{~B}$ & 0.9900 \\
\hline C1-H1A & 1.0000 & C25-C26 & $1.526(9)$ \\
\hline C2-C3 & $1.520(9)$ & $\mathrm{C} 25-\mathrm{H} 25 \mathrm{~A}$ & 1.0000 \\
\hline $\mathrm{C} 2-\mathrm{H} 2 \mathrm{~A}$ & 0.9900 & C26-C27 & $1.533(9)$ \\
\hline C2-H2B & 0.9900 & $\mathrm{C} 26-\mathrm{H} 26 \mathrm{~A}$ & 0.9900 \\
\hline C3-C4 & $1.554(9)$ & $\mathrm{C} 26-\mathrm{H} 26 \mathrm{~B}$ & 0.9900 \\
\hline C3-H3A & 0.9900 & C27-C28 & $1.543(8)$ \\
\hline C3-H3B & 0.9900 & $\mathrm{C} 27-\mathrm{H} 27 \mathrm{~A}$ & 0.9900 \\
\hline C4-H4A & 0.9900 & $\mathrm{C} 27-\mathrm{H} 27 \mathrm{~B}$ & 0.9900 \\
\hline C4-H4B & 0.9900 & C28-H28A & 0.9900 \\
\hline C5-C6 & $1.546(8)$ & $\mathrm{C} 28-\mathrm{H} 28 \mathrm{~B}$ & 0.9900 \\
\hline C5-H5A & 1.0000 & C29-C30 & $1.536(8)$ \\
\hline C6-C7 & $1.519(9)$ & C29-C32 & $1.539(9)$ \\
\hline C6-H6A & 0.9900 & C29-C31 & $1.543(8)$ \\
\hline C6-H6B & 0.9900 & C30-H30A & 0.9800 \\
\hline C7-C8 & $1.539(8)$ & C30-H30B & 0.9800 \\
\hline C7-H7A & 0.9900 & $\mathrm{C} 30-\mathrm{H} 30 \mathrm{C}$ & 0.9800 \\
\hline C7-H7B & 0.9900 & C31-H31A & 0.9800 \\
\hline C8-H8A & 0.9900 & C31-H31B & 0.9800 \\
\hline C8-H8B & 0.9900 & $\mathrm{C} 31-\mathrm{H} 31 \mathrm{C}$ & 0.9800 \\
\hline C9-C12 & $1.520(9)$ & C32-H32A & 0.9800 \\
\hline C9-C11 & $1.543(8)$ & C32-H32B & 0.9800 \\
\hline C9-C10 & $1.552(8)$ & $\mathrm{C} 32-\mathrm{H} 32 \mathrm{C}$ & 0.9800 \\
\hline C10-H10A & 0.9800 & C33-C34 & $1.507(8)$ \\
\hline C10-H10B & 0.9800 & C33-C35 & $1.530(8)$ \\
\hline C10-H10C & 0.9800 & C33-C36 & $1.537(8)$ \\
\hline C11-H11A & 0.9800 & C34-H34A & 0.9800 \\
\hline C11-H11B & 0.9800 & C34-H34B & 0.9800 \\
\hline $\mathrm{C} 11-\mathrm{H} 11 \mathrm{C}$ & 0.9800 & C34-H34C & 0.9800 \\
\hline C12-H12A & 0.9800 & C35-H35A & 0.9800 \\
\hline C12-H12B & 0.9800 & C35-H35B & 0.9800 \\
\hline $\mathrm{C} 12-\mathrm{H} 12 \mathrm{C}$ & 0.9800 & C35-H35C & 0.9800 \\
\hline C13-C14 & $1.524(9)$ & C36-H36A & 0.9800 \\
\hline C13-C15 & $1.530(8)$ & C36-H36B & 0.9800 \\
\hline C13-C16 & $1.535(8)$ & C36-H36C & 0.9800 \\
\hline C14-H14A & 0.9800 & $\mathrm{O} 1-\mathrm{C} 42$ & $1.257(8)$ \\
\hline C14-H14B & 0.9800 & $\mathrm{O} 2-\mathrm{C} 44$ & $1.244(7)$ \\
\hline
\end{tabular}




\begin{tabular}{|c|c|c|c|}
\hline C41-C42 & $1.390(10)$ & C4-C3-H3A & 110.3 \\
\hline C41-C44 & $1.399(9)$ & C2-C3-H3B & 110.3 \\
\hline C41-H41A & 0.9500 & C4-C3-H3B & 110.3 \\
\hline C42-C43 & $1.506(10)$ & H3A-C3-H3B & 108.6 \\
\hline C43-H43A & 0.9800 & C3-C4-P1 & $106.0(4)$ \\
\hline C43-H43B & 0.9800 & C3-C4-H4A & 110.5 \\
\hline C43-H43C & 0.9800 & P1-C4-H4A & 110.5 \\
\hline C44-C45 & $1.529(9)$ & C3-C4-H4B & 110.5 \\
\hline C45-H45A & 0.9800 & P1-C4-H4B & 110.5 \\
\hline C45-H45B & 0.9800 & H4A-C4-H4B & 108.7 \\
\hline \multirow{2}{*}{$\mathrm{C} 45-\mathrm{H} 45 \mathrm{C}$} & 0.9800 & C1-C5-C6 & $114.3(5)$ \\
\hline & & C1-C5-P2 & $113.3(4)$ \\
\hline P4-Rh-P1 & $97.28(5)$ & C6-C5-P2 & $105.7(4)$ \\
\hline P4-Rh-P2 & $155.03(6)$ & C1-C5-H5A & 107.8 \\
\hline P1-Rh-P2 & $83.12(5)$ & C6-C5-H5A & 107.8 \\
\hline P4-Rh-P3 & $83.71(5)$ & P2-C5-H5A & 107.8 \\
\hline P1-Rh-P3 & $155.40(6)$ & C7-C6-C5 & $109.4(5)$ \\
\hline P2-Rh-P3 & $106.23(5)$ & C7-C6-H6A & 109.8 \\
\hline C4-P1-C1 & $93.9(3)$ & C5-C6-H6A & 109.8 \\
\hline C4-P1-C9 & $104.0(3)$ & C7-C6-H6B & 109.8 \\
\hline C1-P1-C9 & 107.1(3) & C5-C6-H6B & 109.8 \\
\hline C4-P1-Rh & $124.70(19)$ & H6A-C6-H6B & 108.3 \\
\hline C1-P1-Rh & $113.08(18)$ & C6-C7-C8 & $106.9(5)$ \\
\hline C9-P1-Rh & 111.78(18) & C6-C7-H7A & 110.4 \\
\hline C8-P2-C13 & $106.0(3)$ & C8-C7-H7A & 110.4 \\
\hline C8-P2-C5 & $93.8(3)$ & C6-C7-H7B & 110.4 \\
\hline C13-P2-C5 & 103.1(3) & C8-C7-H7B & 110.4 \\
\hline C8-P2-Rh & $126.0(2)$ & H7A-C7-H7B & 108.6 \\
\hline C13-P2-Rh & $112.2(2)$ & C7-C8-P2 & $106.3(4)$ \\
\hline C5-P2-Rh & 112.24(19) & C7-C8-H8A & 110.5 \\
\hline C24-P3-C29 & $104.0(3)$ & P2-C8-H8A & 110.5 \\
\hline C24-P3-C21 & $93.5(3)$ & C7-C8-H8B & 110.5 \\
\hline C29-P3-C21 & $102.5(3)$ & P2-C8-H8B & 110.5 \\
\hline C24-P3-Rh & $128.9(2)$ & H8A-C8-H8B & 108.7 \\
\hline C29-P3-Rh & $112.3(2)$ & C12-C9-C11 & $109.4(5)$ \\
\hline C21-P3-Rh & $111.53(18)$ & C12-C9-C10 & $108.0(5)$ \\
\hline C28-P4-C25 & $93.9(3)$ & C11-C9-C10 & $108.3(5)$ \\
\hline C28-P4-C33 & 104.6(3) & C12-C9-P1 & $110.1(4)$ \\
\hline C25-P4-C33 & $106.8(3)$ & C11-C9-P1 & $109.1(4)$ \\
\hline C28-P4-Rh & 124.91(19) & C10-C9-P1 & $111.8(4)$ \\
\hline C25-P4-Rh & $113.0(2)$ & C9-C10-H10A & 109.5 \\
\hline C33-P4-Rh & $111.28(18)$ & С9-C10-H10B & 109.5 \\
\hline C2-C1-C5 & $116.1(5)$ & H10A-C10-H10B & 109.5 \\
\hline C2-C1-P1 & 103.1(4) & C9-C10-H10C & 109.5 \\
\hline C5-C1-P1 & $114.4(4)$ & $\mathrm{H} 10 \mathrm{~A}-\mathrm{C} 10-\mathrm{H} 10 \mathrm{C}$ & 109.5 \\
\hline C2-C1-H1A & 107.6 & H10B-C10-H10C & 109.5 \\
\hline C5-C1-H1A & 107.6 & C9-C11-H11A & 109.5 \\
\hline P1-C1-H1A & 107.6 & C9-C11-H11B & 109.5 \\
\hline C3-C2-C1 & $106.6(5)$ & H11A-C11-H11B & 109.5 \\
\hline $\mathrm{C} 3-\mathrm{C} 2-\mathrm{H} 2 \mathrm{~A}$ & 110.4 & C9-C11-H11C & 109.5 \\
\hline C1-C2-H2A & 110.4 & H11A-C11-H11C & 109.5 \\
\hline C3-C2-H2B & 110.4 & H11B-C11-H11C & 109.5 \\
\hline C1-C2-H2B & 110.4 & C9-C12-H12A & 109.5 \\
\hline $\mathrm{H} 2 \mathrm{~A}-\mathrm{C} 2-\mathrm{H} 2 \mathrm{~B}$ & 108.6 & C9-C12-H12B & 109.5 \\
\hline C2-C3-C4 & $107.0(5)$ & $\mathrm{H} 12 \mathrm{~A}-\mathrm{C} 12-\mathrm{H} 12 \mathrm{~B}$ & 109.5 \\
\hline C2-C3-H3A & 110.3 & C9-C12-H12C & 109.5 \\
\hline
\end{tabular}




$\begin{array}{ll}\text { H12A-C12-H12C } & 109.5 \\ \text { H12B-C12-H12C } & 109.5 \\ \text { C14-C13-C15 } & 109.9(5) \\ \text { C14-C13-C16 } & 108.4(5) \\ \text { C15-C13-C16 } & 108.1(5) \\ \text { C14-C13-P2 } & 108.8(4) \\ \text { C15-C13-P2 } & 107.9(4) \\ \text { C16-C13-P2 } & 113.8(5) \\ \text { C13-C14-H14A } & 109.5 \\ \text { C13-C14-H14B } & 109.5 \\ \text { H14A-C14-H14B } & 109.5 \\ \text { C13-C14-H14C } & 109.5 \\ \text { H14A-C14-H14C } & 109.5 \\ \text { H14B-C14-H14C } & 109.5 \\ \text { C13-C15-H15A } & 109.5 \\ \text { C13-C15-H15B } & 109.5 \\ \text { H15A-C15-H15B } & 109.5 \\ \text { C13-C15-H15C } & 109.5 \\ \text { H15A-C15-H15C } & 109.5 \\ \text { H15B-C15-H15C } & 109.5 \\ \text { C13-C16-H16A } & 109.5 \\ \text { C13-C16-H16B } & 109.5 \\ \text { H16A-C16-H16B } & 109.5 \\ \text { C13-C16-H16C } & 109.5 \\ \text { H16A-C16-H16C } & 109.5 \\ \text { H16B-C16-H16C } & 109.5 \\ \text { C25-C21-C22 } & 114.5(5) \\ \text { C25-C21-P3 } & 113.8(4) \\ \text { C22-C21-P3 } & 105.3(4) \\ \text { C25-C21-H21A } & 107.6 \\ \text { C22-C21-H21A } & 107.6 \\ \text { P3-C21-H21A } & 107.6 \\ \text { C23-C22-C21 } & 109.6(5) \\ \text { C23-C22-H22A } & 109.7 \\ \text { C21-C22-H22A } & 109.7 \\ \text { C23-C22-H22B } & 109.7 \\ \text { C21-C22-H22B } & 109.7 \\ \text { H22A-C22-H22B } & 108.2 \\ \text { C22-C23-C24 } & 107.0(5) \\ \text { C22-C23-H23A } & 110.3 \\ \text { C24-C23-H23A } & 110.3 \\ \text { C22-C23-H23B } & 110.3 \\ \text { C24-C23-H23B } & 110.3 \\ \text { H23A-C23-H23B } & 108.6 \\ \text { C23-C24-P3 } & 106.9(4) \\ \text { C23-C24-H24A } & 110.3 \\ \text { P3-C24-H24A } & 110.3 \\ \text { C23-C24-H24B } & 110.3 \\ \text { P3-C24-H24B } & 110.3 \\ \text { H24A-C24-H24B } & 108.6 \\ \text { C26-C25-C21 } & 115.2(5) \\ \text { C26-C25-P4 } & 103.4(4) \\ \text { C21-C25-P4 } & \\ \text { C26-C25-H25A } & \\ \text { C21-C25-H25A } & \\ \text { P4-C25-H25A } & \\ & \end{array}$

$\begin{array}{ll}\text { C25-C26-C27 } & 105.8(5) \\ \text { C25-C26-H26A } & 110.6 \\ \text { C27-C26-H26A } & 110.6 \\ \text { C25-C26-H26B } & 110.6 \\ \text { C27-C26-H26B } & 110.6 \\ \text { H26A-C26-H26B } & 108.7 \\ \text { C26-C27-C28 } & 107.7(5) \\ \text { C26-C27-H27A } & 110.2 \\ \text { C28-C27-H27A } & 110.2 \\ \text { C26-C27-H27B } & 110.2 \\ \text { C28-C27-H27B } & 110.2 \\ \text { H27A-C27-H27B } & 108.5 \\ \text { C27-C28-P4 } & 106.0(4) \\ \text { C27-C28-H28A } & 110.5 \\ \text { P4-C28-H28A } & 110.5 \\ \text { C27-C28-H28B } & 110.5 \\ \text { P4-C28-H28B } & 110.5 \\ \text { H28A-C28-H28B } & 108.7 \\ \text { C30-C29-C32 } & 108.5(5) \\ \text { C30-C29-C31 } & 107.9(5) \\ \text { C32-C29-C31 } & 109.5(5) \\ \text { C30-C29-P3 } & 113.8(5) \\ \text { C32-C29-P3 } & 109.0(4) \\ \text { C31-C29-P3 } & 108.1(4) \\ \text { C29-C30-H30A } & 109.5 \\ \text { C29-C30-H30B } & 109.5 \\ \text { H30A-C30-H30B } & 109.5 \\ \text { C29-C30-H30C } & 109.5 \\ \text { H30A-C30-H30C } & 109.5 \\ \text { H30B-C30-H30C } & 109.5 \\ \text { C29-C31-H31A } & 109.5 \\ \text { C29-C31-H31B } & 109.5 \\ \text { H31A-C31-H31B } & 109.5 \\ \text { C29-C31-H31C } & 109.5 \\ \text { H31A-C31-H31C } & 109.5 \\ \text { H31B-C31-H31C } & 109.5 \\ \text { C29-C32-H32A } & 109.5 \\ \text { C29-C32-H32B } & 109.5 \\ \text { H32A-C32-H32B } & 109.5 \\ \text { C29-C32-H32C } & 109.5 \\ \text { H32A-C32-H32C } & 109.5 \\ \text { H32B-C32-H32C } & 109.5 \\ \text { C34-C33-C35 } & 110.0(5) \\ \text { C34-C33-C36 } & 109.2(5) \\ \text { C35-C33-C36 } & 107.7(5) \\ \text { C34-C33-P4 } & 109.6(4) \\ \text { C35-C33-P4 } & 108.4(4) \\ \text { C36-C33-P4 } & 111.9(4) \\ \text { C33-C34-H34A } & 109.5 \\ \text { C33-C34-H34B } & 109.5 \\ \text { H34A-C34-H34B } & 109.5 \\ \text { C33-C34-H34C } & 109.5 \\ \text { H34A-C34-H34C } & \\ \text { H34B-C34-H34C } & \\ \text { C33-C35-H35A } & \\ \text { C33-C35-H35B } & \\ & \end{array}$




\begin{tabular}{ll} 
H35A-C35-H35B & 109.5 \\
C33-C35-H35C & 109.5 \\
H35A-C35-H35C & 109.5 \\
H35B-C35-H35C & 109.5 \\
C33-C36-H36A & 109.5 \\
C33-C36-H36B & 109.5 \\
H36A-C36-H36B & 109.5 \\
C33-C36-H36C & 109.5 \\
H36A-C36-H36C & 109.5 \\
H36B-C36-H36C & 109.5 \\
C42-C41-C44 & $129.5(6)$ \\
C42-C41-H41A & 115.2 \\
C44-C41-H41A & 115.2 \\
O1-C42-C41 & $121.9(7)$ \\
O1-C42-C43 & $116.7(7)$ \\
C41-C42-C43 & $121.4(6)$ \\
C42-C43-H43A & 109.5 \\
C42-C43-H43B & 109.5 \\
H43A-C43-H43B & 109.5 \\
C42-C43-H43C & 109.5 \\
H43A-C43-H43C & 109.5 \\
H43B-C43-H43C & 109.5 \\
O2-C44-C41 & $127.7(6)$ \\
O2-C44-C45 & $115.9(6)$ \\
C41-C44-C45 & $116.4(6)$ \\
C44-C45-H45A & 109.5 \\
C44-C45-H45B & 109.5 \\
H45A-C45-H45B & 109.5 \\
C44-C45-H45C & 109.5 \\
H45A-C45-H45C & 109.5 \\
H45B-C45-H45C & 109.5 \\
& \\
\hline
\end{tabular}


Table 9. Anisotropic displacement parameters $\left(\AA^{2} \times 10^{3}\right)$ for $\left.\left[((S, S, R, R)-\text { TangPhos })_{2}\right] \mathrm{Rh}\right][\mathrm{acac}]$. The anisotropic displacement factor exponent takes the form: $-2 \pi^{2}\left[\mathrm{~h}^{2} \mathrm{a}^{* 2} \mathrm{U}^{11}+\ldots+2 \mathrm{~h} \mathrm{k} \mathrm{a} \mathrm{a}^{*} \mathrm{U}^{12}\right]$

\begin{tabular}{|c|c|c|c|c|c|c|}
\hline & $\mathrm{U}^{11}$ & $\mathrm{U}^{22}$ & $\mathrm{U}^{33}$ & $\mathrm{U}^{23}$ & $\mathrm{U}^{13}$ & $\mathrm{U}^{12}$ \\
\hline $\mathrm{Rh}$ & $20(1)$ & 19(1) & $21(1)$ & $-1(1)$ & $1(1)$ & $-1(1)$ \\
\hline $\mathrm{P} 1$ & 23(1) & $22(1)$ & $25(1)$ & $0(1)$ & $-1(1)$ & $-1(1)$ \\
\hline P2 & $25(1$ & $22(1)$ & $22(1)$ & $0(1)$ & $4(1)$ & $-1(1)$ \\
\hline P3 & 23(1) & $25(1)$ & $28(1)$ & $-2(1)$ & $4(1)$ & $-3(1)$ \\
\hline P4 & $22(1)$ & 23(1) & $27(1)$ & $-2(1)$ & 1(1) & $2(1)$ \\
\hline $\mathrm{C} 1$ & $22(2)$ & 34(3) & $32(3)$ & $-10(3)$ & $3(2)$ & $-1(3)$ \\
\hline $\mathrm{C} 2$ & $53(4)$ & $37(4)$ & $36(4)$ & $0(3)$ & 13(3) & $-10(3)$ \\
\hline C3 & $52(4)$ & $32(4)$ & $38(4)$ & $-2(3)$ & 13(3) & $-12(3)$ \\
\hline C4 & 31(3) & 31(3) & $35(3)$ & $3(3)$ & $-1(3)$ & $-4(2)$ \\
\hline C5 & 16(3) & 30(3) & $32(3)$ & $-3(3)$ & $1(2)$ & $1(2)$ \\
\hline C6 & $34(4)$ & $38(4)$ & $40(4)$ & $-1(3)$ & $-4(3)$ & $11(3)$ \\
\hline C7 & 43(4) & 20(3) & $39(4)$ & $0(3)$ & 13(3) & $4(3)$ \\
\hline C8 & $33(3)$ & 30(3) & $33(3)$ & $-7(3)$ & $10(3)$ & $4(3)$ \\
\hline C9 & 29(3) & 41(4) & $17(3)$ & $2(3)$ & $-2(2)$ & $-5(3)$ \\
\hline $\mathrm{C} 10$ & $35(4)$ & $72(5)$ & $41(4)$ & $14(4)$ & $-11(3)$ & $-12(3)$ \\
\hline C11 & $54(4)$ & 30(3) & $29(3)$ & $0(3)$ & $-7(3)$ & $2(3)$ \\
\hline C12 & $33(3)$ & 33(4) & $38(4)$ & $5(3)$ & $-4(3)$ & $4(3)$ \\
\hline $\mathrm{C} 13$ & $28(3)$ & $38(3)$ & $28(3)$ & $-6(3)$ & $-1(2)$ & $-9(3)$ \\
\hline C14 & 39(3) & 42(4) & $32(4)$ & $-1(3)$ & $-1(3)$ & $-6(3)$ \\
\hline $\mathrm{C} 15$ & $33(3)$ & $48(4)$ & $32(4)$ & $-16(3)$ & $2(3)$ & $-3(3)$ \\
\hline C16 & $50(4)$ & 43(4) & $34(4)$ & $-17(3)$ & $8(3)$ & $-4(3)$ \\
\hline $\mathrm{C} 21$ & 20(3) & 30(3) & $34(3)$ & $5(3)$ & $6(2)$ & $0(2)$ \\
\hline C22 & 20(3) & 43(4) & $46(4)$ & $5(3)$ & $2(3)$ & $3(2)$ \\
\hline $\mathrm{C} 23$ & $28(3)$ & $55(4)$ & $44(4)$ & $-1(4)$ & $-3(3)$ & $-4(3)$ \\
\hline C24 & $32(3)$ & 31(3) & $32(4)$ & $-4(3)$ & $-1(3)$ & $-8(3)$ \\
\hline $\mathrm{C} 25$ & 24(3) & $38(4)$ & $29(3)$ & $4(3)$ & $2(3)$ & $5(3)$ \\
\hline C26 & 30(3) & $35(4)$ & $34(4)$ & $-8(3)$ & $7(3)$ & 3(3) \\
\hline $\mathrm{C} 27$ & $35(3)$ & $36(4)$ & $31(3)$ & $-15(3)$ & $0(3)$ & $0(3)$ \\
\hline C28 & 29(3) & 23(3) & $39(4)$ & $-7(3)$ & 1(3) & $4(2)$ \\
\hline C29 & $31(3)$ & 26(3) & $36(3)$ & $0(3)$ & $14(3)$ & $-5(3)$ \\
\hline C 30 & $35(4)$ & $47(4)$ & $49(4)$ & 13(4) & 11(3) & $-9(3)$ \\
\hline C31 & 44(4) & 28(3) & $43(4)$ & $5(3)$ & $6(3)$ & $6(3)$ \\
\hline C32 & 45(4) & $33(4)$ & $35(4)$ & $10(3)$ & $2(3)$ & 4(3) \\
\hline C33 & $32(3)$ & 24(3) & $31(3)$ & $0(3)$ & $-2(3)$ & $8(2)$ \\
\hline C34 & 42(4) & $32(3)$ & $27(3)$ & $3(3)$ & $-11(3)$ & $2(3)$ \\
\hline C35 & $39(4)$ & 32(3) & $35(3)$ & $12(3)$ & $0(3)$ & $-3(3)$ \\
\hline C36 & $37(4)$ & 37(4) & $47(4)$ & $5(3)$ & 1(3) & $12(3)$ \\
\hline $\mathrm{O} 1$ & $66(4)$ & $45(3)$ & $72(4)$ & $-2(3)$ & $28(3)$ & $-11(3)$ \\
\hline $\mathrm{O} 2$ & $52(3)$ & 38(3) & $36(2)$ & $3(2)$ & $2(2)$ & $-5(2)$ \\
\hline C41 & 49(4) & $35(4)$ & 41(4) & $0(3)$ & $4(3)$ & $18(3)$ \\
\hline C42 & 43(4) & $31(3)$ & $39(4)$ & $-9(3)$ & $15(3)$ & $-1(3)$ \\
\hline C43 & $36(3)$ & $59(4)$ & $49(4)$ & $-15(4)$ & $6(3)$ & $-2(3)$ \\
\hline C44 & $36(3)$ & $36(4)$ & $35(3)$ & $-13(3)$ & $-5(3)$ & $4(3)$ \\
\hline C45 & 63(5) & 63(6) & $74(6)$ & $-15(5)$ & $-25(4)$ & 7(4) \\
\hline
\end{tabular}


Table 10. Hydrogen coordinates ( $\left.\mathrm{x} 10^{4}\right)$ and isotropic displacement parameters $\left(\AA^{2} \times 10^{3}\right)$ for $\left.\left[((S, S, R, R) \text {-TangPhos })_{2}\right] \mathrm{Rh}\right][\mathrm{acac}]$.

\begin{tabular}{|c|c|c|c|c|}
\hline & $\mathrm{x}$ & $\mathrm{y}$ & z & $\mathrm{U}(\mathrm{eq})$ \\
\hline H1A & 6416 & 4792 & 1181 & 35 \\
\hline $\mathrm{H} 2 \mathrm{~A}$ & 6555 & 5578 & 225 & 50 \\
\hline $\mathrm{H} 2 \mathrm{~B}$ & 5349 & 5833 & 236 & 50 \\
\hline $\mathrm{H} 3 \mathrm{~A}$ & 6221 & 7085 & 657 & 49 \\
\hline H3B & 6871 & 6455 & 1134 & 49 \\
\hline $\mathrm{H} 4 \mathrm{~A}$ & 4751 & 6985 & 1261 & 39 \\
\hline H4B & 5581 & 6859 & 1824 & 39 \\
\hline H5A & 5470 & 4112 & 177 & 31 \\
\hline H6A & 6583 & 3119 & 620 & 45 \\
\hline Н6B & 6136 & 3259 & 1325 & 45 \\
\hline H7A & 5522 & 1859 & 1021 & 41 \\
\hline H7B & 5199 & 2221 & 327 & 41 \\
\hline H8A & 4161 & 2595 & 1512 & 39 \\
\hline H8B & 3637 & 2251 & 862 & 39 \\
\hline H10A & 6778 & 5133 & 2844 & 74 \\
\hline H10B & 6684 & 5939 & 2340 & 74 \\
\hline $\mathrm{H} 10 \mathrm{C}$ & 6905 & 4933 & 2099 & 74 \\
\hline H11A & 5075 & 5554 & 3292 & 56 \\
\hline H11B & 4067 & 5590 & 2852 & 56 \\
\hline $\mathrm{H} 11 \mathrm{C}$ & 4939 & 6347 & 2781 & 56 \\
\hline $\mathrm{H} 12 \mathrm{~A}$ & 5439 & 3983 & 2922 & 52 \\
\hline H12B & 5513 & 3765 & 2177 & 52 \\
\hline $\mathrm{H} 12 \mathrm{C}$ & 4424 & 4015 & 2489 & 52 \\
\hline H14A & 3344 & 4644 & -798 & 56 \\
\hline H14B & 4384 & 4750 & -394 & 56 \\
\hline $\mathrm{H} 14 \mathrm{C}$ & 3326 & 5165 & -130 & 56 \\
\hline H15A & 1927 & 3624 & -389 & 57 \\
\hline H15B & 1943 & 4160 & 272 & 57 \\
\hline $\mathrm{H} 15 \mathrm{C}$ & 2095 & 3088 & 265 & 57 \\
\hline H16A & 3508 & 3013 & -852 & 63 \\
\hline H16B & 3677 & 2425 & -222 & 63 \\
\hline $\mathrm{H} 16 \mathrm{C}$ & 4580 & 3068 & -475 & 63 \\
\hline $\mathrm{H} 21 \mathrm{~A}$ & 560 & 5147 & 2528 & 34 \\
\hline $\mathrm{H} 22 \mathrm{~A}$ & -879 & 5467 & 1947 & 43 \\
\hline $\mathrm{H} 22 \mathrm{~B}$ & -244 & 5609 & 1299 & 43 \\
\hline $\mathrm{H} 23 \mathrm{~A}$ & -1031 & 4232 & 1176 & 51 \\
\hline $\mathrm{H} 23 \mathrm{~B}$ & -727 & 3921 & 1885 & 51 \\
\hline $\mathrm{H} 24 \mathrm{~A}$ & 629 & 4044 & 803 & 38 \\
\hline H24B & 542 & 3218 & 1296 & 38 \\
\hline $\mathrm{H} 25 \mathrm{~A}$ & 708 & 6680 & 1894 & 36 \\
\hline H26A & 1995 & 6146 & 2945 & 39 \\
\hline $\mathrm{H} 26 \mathrm{~B}$ & 945 & 6721 & 2995 & 39 \\
\hline $\mathrm{H} 27 \mathrm{~A}$ & 2505 & 7673 & 2990 & 41 \\
\hline $\mathrm{H} 27 \mathrm{~B}$ & 1690 & 7945 & 2446 & 41 \\
\hline $\mathrm{H} 28 \mathrm{~A}$ & 3630 & 7058 & 2277 & 37 \\
\hline $\mathrm{H} 28 \mathrm{~B}$ & 3106 & 7791 & 1813 & 37 \\
\hline H30A & 791 & 2816 & 3041 & 66 \\
\hline H30B & 326 & 2897 & 2337 & 66 \\
\hline $\mathrm{H} 30 \mathrm{C}$ & 230 & 3726 & 2821 & 66 \\
\hline H31A & 2453 & 2369 & 2590 & 57 \\
\hline
\end{tabular}




\begin{tabular}{lrrrr} 
H31B & 3058 & 3008 & 2102 & 57 \\
H31C & 2044 & 2481 & 1875 & 57 \\
H32A & 2312 & 3684 & 3359 & 56 \\
H32B & 1820 & 4616 & 3122 & 56 \\
H32C & 2922 & 4301 & 2859 & 56 \\
H34A & 1295 & 6512 & -3 & 51 \\
H34B & 858 & 6018 & 619 & 51 \\
H34C & 1930 & 5702 & 313 & 51 \\
H35A & 2860 & 7491 & 77 & 53 \\
H35B & 3483 & 6678 & 399 & 53 \\
H35C & 3402 & 7631 & 754 & 53 \\
H36A & 1194 & 7991 & 509 & 61 \\
H36B & 1742 & 8161 & 1182 & 61 \\
H36C & 733 & 7541 & 1140 & 61 \\
H41A & 7854 & 4024 & -293 & 50 \\
H43A & 5649 & 4301 & -1635 & 72 \\
H43B & 6745 & 4718 & -1826 & 72 \\
H43C & 6045 & 5207 & -1301 & 72 \\
H45A & 9530 & 5843 & -448 & 100 \\
H45B & 9624 & 4777 & -340 & 100 \\
H45C & 8909 & 5369 & 120 & 100 \\
& & & & \\
\hline
\end{tabular}

